\title{
Full-Field/Scattered-Field Formulation Containing a Dielectric Interface
}

\author{
David N. Smithe*
}

Tech-X Corporation 5621 Arapahoe Ave. Suite A Boulder, CO 80303, USA

\begin{abstract}
The usual full-field/scattered-field formulation is generalized to include a planar dielectric interface passing through the space. In this case, the source condition surrounding the central full-field region emits and captures waves consistent with scattering from a planar dielectric interface. Thus the wave in the outer scattered-field region represents just the part of the scattered wave that is due to non-planar dielectric or conductor geometry. The formulation is exact to machine precision, regardless of wavelength being smaller or larger than the simulation domain, and includes the exact second-order correction to the nominal scattering coefficients due to finite-difference truncation error. The method works even in the case of total internal reflection at the dielectric interface, that is, with the transmitted side exhibiting exponential decay rather than a propagating wave. A simple variation allows the dielectric to be a plasma, with correct scattering, regardless of whether the plasma is under-dense or over-dense. Possible applications for this facility range from RCS-type computations at hydrological or atmospheric interfaces, to geometry characterization or damage assessment of surfaces, to general boundary conditions for simulations of intermediate size EM phenomenon, e.g., $L \sim \lambda$, to finally, an algorithm test-bed for the subject of cut-cell dielectric in the finite-difference representation.
\end{abstract}

Keywords: Scattered-field, RCS (radar cross section), dielectric, finite-difference, cut-cell, plasma, metamaterial, VORPAL.

\section{INTRODUCTION}

The principle motivation for this work was the desire for an electromagnetic wave source condition in a simulation that is on-the-order-of, or smaller than a wavelength. Generally speaking, the simulation region constitutes a magnified view of a portion of a larger physics problem containing several wavelengths, and qualitative propagating wave behavior. Because the simulation size is smaller than a wavelength, periodic boundaries cannot be used, and also due to the ultimate desire to include charged particles and non-linear physics, a Floquet-type boundary is also not ideal, as this would require complex-value fields, particle weights, and a means of interpreting the non-linear products of such.

One suggestion for treating this difficult source problem is to use a seldom-realized property of the fullfield/scattered-field technique [1]. This approach is primarily used to look at scattering of an RF wave from an object, in a simulation domain many wavelengths across. However, the full-field/scattered-field source condition contains no restriction on wavelength, and indeed, works equally well for wavelengths larger than the simulation domain. It is this property we wished to take advantage of in this work. Fig. (1) shows how microscopic scale simulation with the fullfield/scattered-field technique is possible.

The "EM-microscope" simulation contains yet one more important feature. We wished to get a magnified view of the physical behavior in the vicinity of a dielectric interface. The interface could be between two solid dielectrics, between dielectric and vacuum, or between dielectric and plasma. Thus, we required the full-field/scattered-field technique to

*Address correspondence to this author at the Tech-X Corporation 5621 Arapahoe Ave. Suite A Boulder, CO 80303, USA; Tel: 303-996-2023; Fax: 303-448-7756; Email: smithe@txcorp.com be generalized to include scattering from a planar dielectric interface contained within the problem.

In this paper, we do not report on the microscopic RF simulations themselves, but rather the generalization of the full-field/scattered-field technique to include the feature of a dielectric interface. It is recognized that this capability likely reaches beyond its original purpose, and constitutes an important simulation result in its own right, applicable to several different areas, including traditional scattering type simulations, and the general problem of simulating arbitrary geometry dielectrics, and meta-materials.

Our implementation of the full-field/scattered-field technique follows that of Reference [1], using a Yee-cell / leap-frog differencing strategy [2]. However, in order to treat the dielectric interface within the confines of the technique, the source conditions must emit/reabsorb not only the incident wave but also the reflected and transmitted wave due to the interface. This augmentation is the key result of this paper. Successful use of the technique requires that the propagation characteristics of the incident, transmitted, and reflected waves must be known exactly, for the finitedifference implementation, not just for the analogous continuum problem. The dispersive corrections for light in a discrete simulation are indeed well known, and used effectively here. However, in addition, there are discretization corrections to the scattering coefficients at a dielectric interface which must also be introduced, and these corrections are derived in this paper, and demonstrated in simulation.

This paper illustrates the use of a simple analytic treatment for a cold-unmagnetized-plasma as one of the dielectric components, following a technique from Reference [3]. This treatment includes an internal field variable, the plasma current in this case, and serves as an example on how 
(a)

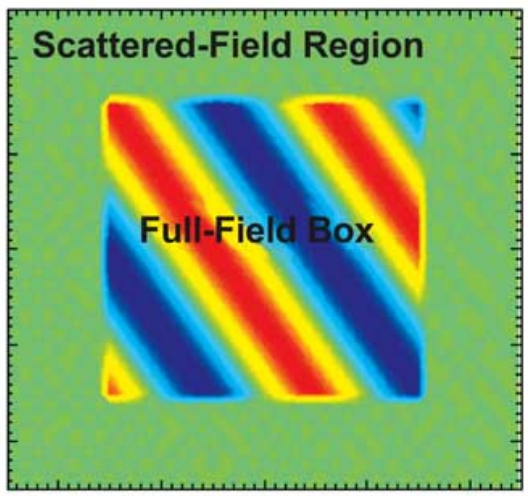

(b)

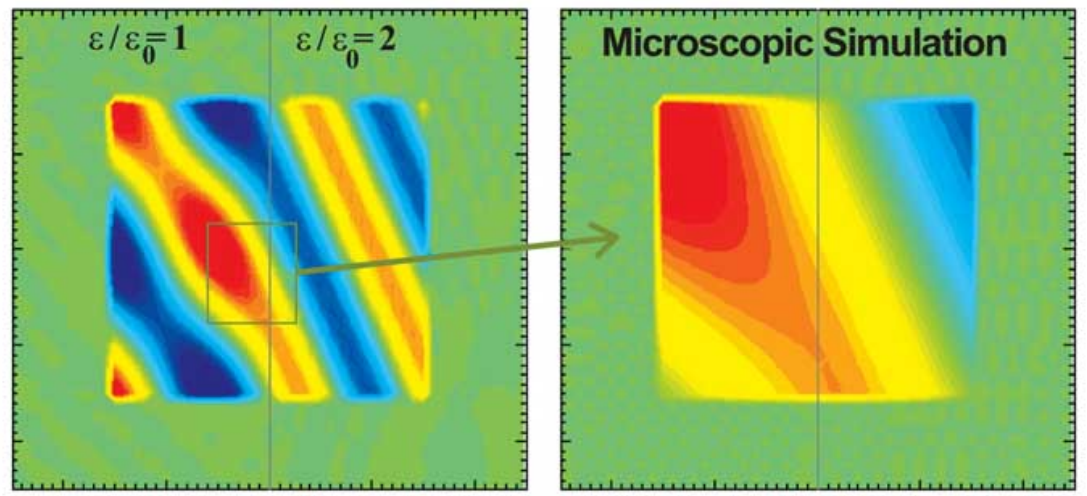

Fig. (1). Illustration of this paper's evolution of the full-field/scattered-field technique. (a) typical simulation where the source condition emits and reabsorbs the incident plane wave, so that it does not appear in the scattered-field region, (b) generalization to include dielectric interface and transmitted and reflected waves, and (c) demonstration of case where simulation dimensions are shorter than a wavelength, e.g., an EM microscopic simulation.

meta-materials in general, with internal resonances or other physical attributes, can be model using the FDTD approach.

Finally, the fact that the full-field/scattered-field technique utilizes an exact solution for the discrete problem, rather than relying on convergence analysis, makes it an interesting and novel tool for the study of how dielectric interfaces behave in an FDTD simulation. For example in this work it was seen how the loss of continuity, due to discreteness, can counter the anticipated loss in wellcentering at the interface plane, resulting in a second-order accurate solution, even when one is not necessarily expected.

We note that in this paper, the symbol for the dielectric, $\varepsilon$, represents the absolute permittivity, with units of Farads/meter. Thus, $\varepsilon / \varepsilon_{0}$ denotes the relative permittivity.

\section{FULL-FIELD/SCATTERED-FIELD TECHNIQUE}

The full-field/scattered-field technique is well described and documented elsewhere, for example Reference [1]. The full-field/scattered-field technique is perhaps most easily understood in 1-D. Any localized source, either electric or magnetic current, will propagate a wave equally in both directions. However, if two closely spaced localized sources, of appropriate phase shift, are used, the result of superposition can be a single wave propagating in only one direction from the source-pair. One may then place another source-pair some distance from the first, to emit a similar uni-direction wave that exactly cancels the first, as it passes by, resulting in a wave which appears to be emitted at one source-pair, then travels in one direction towards the second source-pair, and then appears to be re-absorbed. This process is illustrated in Fig. (2).

Obviously, in order for this to happen, one must have exact a priori knowledge of the propagating wave's frequency, wavenumber, amplitude, and phase, in order to achieve the desired illusion of re-absorption. This explains the emphasis of the following sections where we shall seek
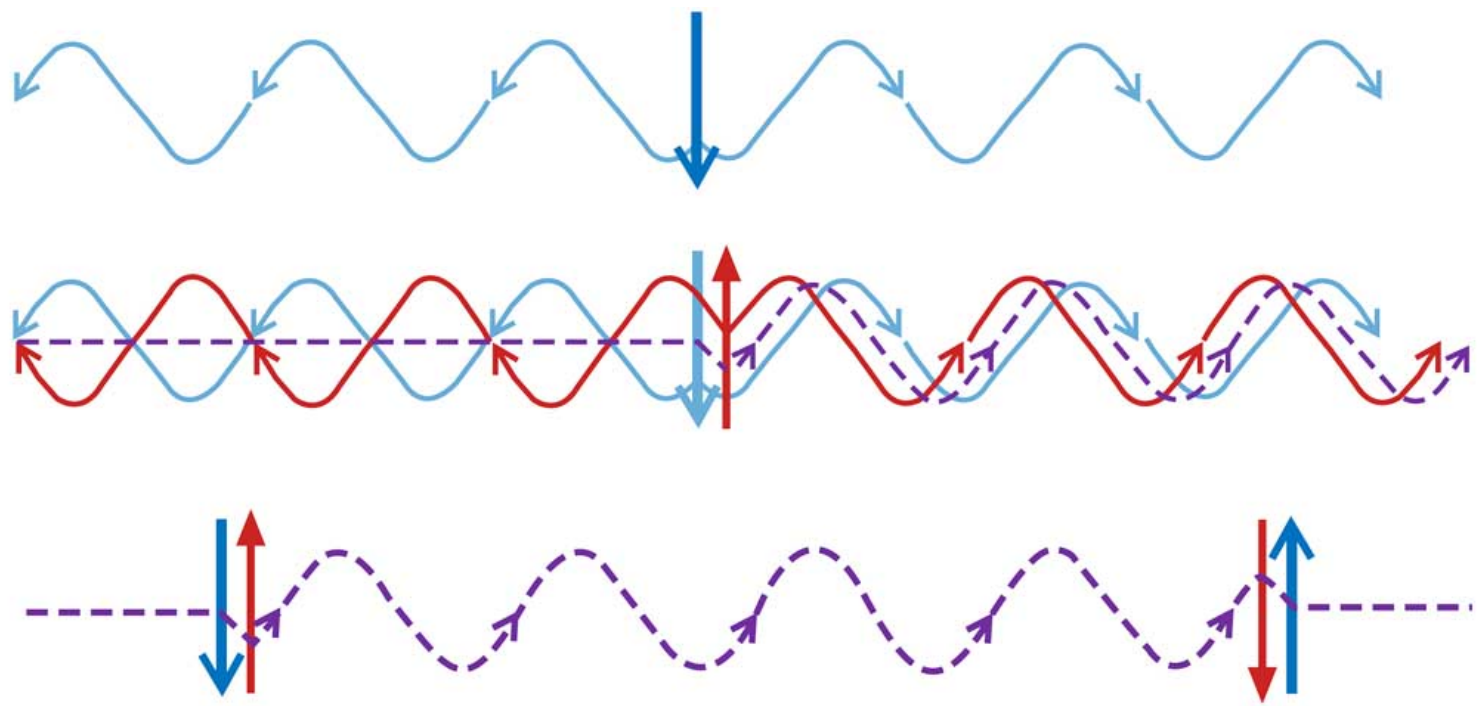

Fig. (2). Use of localized sources to produce a full-field/scattered-field simulation. Top shows a single localized source which propagates equally to the left and right (blue line). Middle shows how the superposition of two such closely spaced sources (blue and red lines) can produce a net wave (dotted line) appearing to propagate in only one direction. And bottom shows how two such source-pairs can appear to produce a uni-directional wave that is emitted at one source-pair and reabsorbed at a second source-pair. 
the exact wavenumber, and scattering coefficients in the discrete version of Maxwell.

\section{ANALYSIS OF PLANE WAVE SCATTERING IN FINITE DIFFERENCE}

This section derives the usual forms for scattering coefficients in continuum, and also their subtly different discrete analogues, using a formalism that shares a common waveform between the continuum and discrete versions of the same problem. An important point is that whereas field continuity alone is sufficient to derive the scattering coefficients for a continuum, this concept of continuity partly breaks-down for the discrete analogue. Instead, one we introduce the quadratic conservation theorems, either continuum, or discrete, in order to complete the analysis.

\subsection{Continuum Plane-Wave}

For a continuum with dielectric $\varepsilon$, the plane wave solution to Maxwell's equations is well known, and is analytic, with notation as follows. Assume a plane wave characterized by real frequency, $\omega$, possibly complex electric field polarization vector, $\mathbf{e}_{E}$, and possibly complex wavenumber, $\mathbf{k}$, such that $\mathbf{e}_{E} \cdot \mathbf{k}=0$. The magnitude of the wavenumber, $\mathbf{k} \cdot \mathbf{k}$, is constrained by the dispersion relation.

The electric field polarization direction, $\mathbf{e}_{E}$, has the generality of a two-dimensional space, normal to $\mathbf{k}$. Introduce the dielectric interface's normal vector, $\mathbf{e}_{x}$, and employ it to separate the two polarization directions. One of these polarization directions will be in the plane of the dielectric interface, e.g., define $\mathbf{e}_{\|}$in the direction of $\mathbf{e}_{x} \times \mathbf{k}$, and the other normal to that, e.g., in the direction of $\mathbf{e}_{\|} \times \mathbf{k}$. The analysis now proceeds separately for each of the two polarization scenarios, one called TE, in which $\mathbf{e}_{E}=\mathbf{e}_{\|}$and the other, called TM, in which $\mathbf{e}_{E}$ is parallel to $\mathbf{e}_{\|} \times \mathbf{k}$. (For the case of exact normal incidence, e.g., $\mathbf{e}_{x} \times \mathbf{k}=0$, take $\mathbf{e}_{\|}=\mathbf{e}_{E}$, that is, exact normal incidence is treated within the TE scenario.) Thus, an arbitrary polarization state should be considered to be a superposition of these two states, with possibly complex valued superposition coefficients. The electric and magnetic fields, for a plane wave can thus be written

$$
\begin{aligned}
& \mathbf{E}(\mathbf{x}, t)=\operatorname{Real}\left\{\left[\alpha_{T E} \omega \mathbf{e}_{\|}+\alpha_{T M}\left(\mathbf{e}_{\|} \times \mathbf{k}\right)\right] \psi(\varphi(\mathbf{x}, t))\right\} \\
& \mathbf{B}(\mathbf{x}, t)=\operatorname{Real}\left\{\left[\alpha_{T E}\left(\mathbf{k} \times \mathbf{e}_{\|}\right)+\alpha_{T M}\left(\omega \mu_{0} \varepsilon\right) \mathbf{e}_{\|}\right] \psi(\varphi(\mathbf{x}, t))\right\}
\end{aligned}
$$

where the dispersion relation, complex waveform, and wavefront-phase are

$$
\begin{aligned}
& \mathbf{k} \cdot \mathbf{k}=\omega^{2} \varepsilon \mu_{0} \\
& \psi(s)=\mathrm{e}^{i s} U(s) \\
& \varphi(\mathbf{x}, t)=\mathbf{k} \cdot\left(\mathbf{x}-\mathbf{x}_{0}\right)-\omega t
\end{aligned}
$$

and where $\mathbf{x}_{0}$ is some point well removed from the simulation domain in the $-\mathbf{k}_{\text {incident }}$ direction, and $U(s)$ is some suitable smooth turn-on function, which is zero for $s \leq 0$, and goes to unity after $s$ exceeds a reasonable number of oscillation cycles.

The cycle-averaged Poynting flow, $\mathbf{P}=\mu_{0}{ }^{-1} \mathbf{E} \times \mathbf{B}$, for this plane wave is

$\mathbf{P}(\mathbf{x}, t)=1 / 2 \mu_{0}^{-1}|\psi(\varphi(\mathbf{x}, t))|^{2}\left(\left|\alpha_{T E}\right|^{2}+\mu_{0} \varepsilon\left|\alpha_{T M}\right|^{2}\right) \omega \operatorname{Real}\{\mathbf{k}\}$

\subsection{Discrete Plane Wave}

Assume the Yee-cell spatial discretization of electric and magnetic fields, and leap-frog temporal discretization. There is a similar exact plane-wave solution for this discrete Maxwell problem, assuming that the time is well past the turn-on-transient. As with the continuum case, assume a real frequency, $\omega$, a complex electric field polarization direction, $\mathbf{e}_{E}$, and complex wavenumber, $\mathbf{k}$, satisfying the continuum dispersion relation, $\mathbf{k} \cdot \mathbf{k}=\omega^{2} \varepsilon \mu_{0}$. The fields are given exactly as before, except that the waveform, $\psi(\varphi(\mathbf{x}, t))$, is evaluated at the appropriate discrete position and times of the Yee-cell / leap-frog system, and this waveform is a function of a "finite-difference" wave-front-phase,

$\varphi(\mathbf{x}, t)=\mathbf{k} \cdot\left(\mathbf{x}-\mathbf{x}_{0}\right)-\omega t$

which is based upon, $\mathbf{k}$, a "finite-difference-wavenumber". (Finite-difference quantities are indicated with a doublestrike-through.) This quantity, $\mathbf{k}$, is slightly modified from $\mathbf{k}$, in each mesh direction, $\mathbf{e}_{x}, \mathbf{e}_{y}$, and $\mathbf{e}_{z}$, by the mesh sizes, $\delta x$, $\delta y$, and $\delta z$, and the finite time-step, $\delta t$, that is, specifically,

$\mathbf{k} \cdot \mathbf{e}_{x} \equiv(2 / \delta x) \sin ^{-1}\left(\left(1 / 2 \mathbf{k} \cdot \mathbf{e}_{x} \delta x\right) \operatorname{sinc}(1 / 2 \omega \delta t)\right)$

and similar for $\mathbf{k} \cdot \mathbf{e}_{y}$ and $\mathbf{k} \cdot \mathbf{e}_{z}$, where the sinc-function is defined as usual, e.g., $\operatorname{sinc}(s) \equiv \sin (s) / s$. This difference means that the actual direction of propagation of the wave fronts is not precisely along $\mathbf{k}$, nor is the speed exactly $\left(\varepsilon \mu_{0}\right)^{-1 / 2}$, but rather something close to these, assuming reasonable spatial resolution of the wavelength.

The Poynting flow in a Yee-cell leap-frog finitedifference EM simulation is an oft-misunderstood quantity, as an exact form, and its connection to continuum Poynting flux can be difficult to interpret, especially since components of $\mathbf{E}$ and $\mathbf{B}$, are never at the same spatial location and time. However, in pure mathematical terms, the Yee-cell/leap-frog Maxwell system is in the general category of difference equations, and thus exhibits a volume-integrated quadratic conservation theorem of its own right, independent of its continuum analogue. This conservation theorem, which is detailed in Reference [4], contains a highly useful version of finite-difference Poynting flux. For example, if the Poyntingintegration volume has a full-grid $y-z$ plane as its bounding surface, then the flux, centered at the half-time-steps, through a cell-face, consists of the average of the 16 possible combinations of $E_{y} \times B_{z}$ and $E_{z} \times B_{y}$, which have $\mathbf{E}$ components surrounding the cell-face, half a time-step before or after, and corresponding $\mathbf{B}$ components half a cell above or below the plane. The result is that the time-averaged finitedifference Poynting flow of the plane wave is

$$
\begin{gathered}
\mathbf{P}(\mathbf{x}, t) \cdot \mathbf{e}_{x}=1 / 2 \mu_{0}^{-1}|\psi(\varphi(\mathbf{x}, t))|^{2} \cos (1 / 2 \omega \delta t) \times \\
\cos \left(1 / 2 \mathbf{k} \cdot \mathbf{e}_{x} \delta x\right)\left(\left|\alpha_{T E}\right|^{2}+\mu_{0} \varepsilon\left|\alpha_{T M}\right|^{2}\right) \omega \mathbf{k} \cdot \mathbf{e}_{x} \\
=1 / 4 \mu_{0}^{-1}|\psi(\varphi(\mathbf{x}, t))|^{2}\left(\left|\alpha_{T E}\right|^{2}+\mu_{0} \varepsilon\left|\alpha_{T M}\right|^{2}\right) \times \\
(\delta t / \delta x) \omega^{2} \sin \left(\mathbf{k} \cdot \mathbf{e}_{x} \delta x\right) / \tan (1 / 2 \omega \delta t)
\end{gathered}
$$

where some algebra has been performed to get the second line purely in terms of $\mathbf{k}$, instead of a mixture of $\mathbf{k}$ and $\mathbf{k}$. The " $\cos \left(1 / 2 \mathbf{k} \cdot \mathbf{e}_{x} \delta x\right)$ " factor in the first line is the critical new aspect for the discrete problem, as this factor introduces an additional dependence on incident vs transmitted wavenumber, which has no analogue in a continuum. 


\subsection{Continuum Scattering Coefficients}

Let the dielectrics on the incident/transmitted side be $\varepsilon_{i n c}$ and $\varepsilon_{t r n}$. Require that the incident wave is propagating, e.g., that $\mathbf{k}_{\text {inc }}$, is pure real. Then the reflected and transmitted wavenumbers are

$$
\begin{aligned}
& \mathbf{k}_{r e f}=\mathbf{k}_{i n c} \cdot\left[\mathbb{1}-\mathbf{e}_{x} \mathbf{e}_{x}\right]-\mathbf{k}_{i n c} \cdot\left[\mathbf{e}_{x} \mathbf{e}_{x}\right] \\
& \mathbf{k}_{t r m}=\mathbf{k}_{i n c} \cdot\left[\mathbb{1}-\mathbf{e}_{x} \mathbf{e}_{x}\right]+\mathbf{k}_{i n c} \cdot\left[\mathbf{e}_{x} \mathbf{e}_{x}\right]\left(\left(\mathbf{e}_{k} \cdot \mathbf{e}_{x}\right)^{2}+\left(\varepsilon_{i r n} / \varepsilon_{i n c}\right)-1\right)^{1 / 2} /\left(\mathbf{e}_{k} \mathbf{e}_{x}\right) \\
& \quad=\mathbf{k}_{i n c} \cdot\left[\mathbb{1}-\mathbf{e}_{x} \mathbf{e}_{x}\right]+i \mathbf{k}_{i n c} \cdot\left[\mathbf{e}_{x} \mathbf{e}_{x}\right]\left(1-\left(\mathbf{e}_{k} \cdot \mathbf{e}_{x}\right)^{2}-\left(\varepsilon_{t r n} / \boldsymbol{\varepsilon}_{i n c}\right)\right)^{1 / 2} /\left(\mathbf{e}_{k} \mathbf{e}_{x}\right)
\end{aligned}
$$

Total internal reflection occurs if taking the square-root of a negative number in the first form for $\mathbf{k}_{t r n}$, in which case, the alternate complex-form should be used. The incident, reflected and transmitted wave front phases, the latter possibly being complex-valued, are

$$
\begin{aligned}
& \varphi_{i n c}(\mathbf{x}, t)=\mathbf{k}_{i n c} \cdot\left(\mathbf{x}-\mathbf{x}_{0}\right)-\omega t \\
& \varphi_{r e f}(\mathbf{x}, t)=\mathbf{k}_{r e f} \cdot\left(\mathbf{x}-\mathbf{x}_{0}\right)-\omega t+\left(\mathbf{k}_{r e f}-\mathbf{k}_{i n c}\right) \cdot\left[\mathbf{e}_{x} \mathbf{e}_{x}\right] \cdot\left(\mathbf{x}_{s}-\mathbf{x}_{0}\right) \\
& \varphi_{t r n}(\mathbf{x}, t)=\mathbf{k}_{t r n} \cdot\left(\mathbf{x}-\mathbf{x}_{0}\right)-\omega t+\left(\mathbf{k}_{t r n}-\mathbf{k}_{i n c}\right) \cdot\left[\mathbf{e}_{x} \mathbf{e}_{x}\right] \cdot\left(\mathbf{x}_{s}-\mathbf{x}_{0}\right)
\end{aligned}
$$

where $\mathbf{x}_{s}$ is any point on the dielectric interface. These three phases are designed to be equal in value for all points on the dielectric interface so that when field-continuity is imposed there, the waveform part of the field,

$$
\psi\left(\varphi_{\text {inc }}\left(\mathbf{x}_{s}, t\right)\right)=\psi\left(\varphi_{\text {ref }}\left(\mathbf{x}_{s}, t\right)\right)=\psi\left(\varphi_{\text {trn }}\left(\mathbf{x}_{s}, t\right)\right)
$$

is simply a common factor. Finally, introduce unit step functions, $S_{\text {inc }}(\mathbf{x}), S_{\text {ref }}(\mathbf{x})=S_{\text {inc }}(\mathbf{x})$, and $S_{\text {trn }}(\mathbf{x})$, which are unity on their designated half-space, zero on the opposite halfspace, and in order to properly construct the waveforms, have value of $1 / 2$ exactly on the interface.

\subsubsection{TE Scattering in Continuum}

In the TE case, refer back to Equation (1) for the waveforms, set $\alpha_{T M}=0$ for all waves, set $\alpha_{T E}=1$ for the incident wave, and seek $\alpha_{T E}=\rho_{T E}$ for the reflected wave and, $\alpha_{T E}=\tau_{T E}$ for the transmitted wave. The solution over the entire spatial domain will then be of the form

$$
\begin{aligned}
& \mathbf{E}_{\text {analytic }}(\mathbf{x}, t)=\operatorname{Real}\left\{\omega \mathbf{e}_{\|} \psi\left(\varphi_{\text {inc }}(\mathbf{x}, t)\right) S_{\text {inc }}(\mathbf{x})\right. \\
& \quad+\rho_{\mathrm{TE}} \omega \mathbf{e}_{\|} \psi\left(\varphi_{r e f}(\mathbf{x}, t)\right) S_{\text {ref }}(\mathbf{x}) \\
& \left.\quad+\tau_{\mathrm{TE}} \omega \mathbf{e}_{\|} \psi\left(\varphi_{t r n}(\mathbf{x}, t)\right) S_{t r n}(\mathbf{x})\right\} \\
& \mathbf{B}_{\text {analytic }}(\mathbf{x}, t)=\operatorname{Real}\left\{\left(\mathbf{k}_{\text {inc }} \times \mathbf{e}_{\|}\right) \psi\left(\varphi_{\text {inc }}(\mathbf{x}, t)\right) S_{\text {inc }}(\mathbf{x})\right. \\
& \quad+\rho_{\mathrm{TE}}\left(\mathbf{k}_{r e f} \times \mathbf{e}_{\|}\right) \psi\left(\varphi_{r e f}(\mathbf{x}, t)\right) S_{r e f}(\mathbf{x}) \\
& \left.\quad+\tau_{\mathrm{TE}}\left(\mathbf{k}_{t r n} \times \mathbf{e}_{\|}\right) \psi\left(\varphi_{t r n}(\mathbf{x}, t)\right) S_{t r n}(\mathbf{x})\right\}
\end{aligned}
$$

Two conditions are required to constrain the values of $\rho$ and $\tau$, the reflection and transmission coefficients. In the usual derivation, continuity of the in-plane $\mathbf{E}$ and $\mathbf{B}$ components provides the constraints, e.g., taking the dotproduct of $\mathbf{E}$ with $\mathbf{e}_{\|}$and $\mathbf{B}$ with $\left(\mathbf{e}_{x} \times \mathbf{e}_{\|}\right)$, will give

$1+\rho_{\mathrm{TE}}=\tau_{\mathrm{TE}}$

$\left(\mathbf{k}_{i n c} \cdot \mathbf{e}_{x}\right)+\rho_{\mathrm{TE}}\left(\mathbf{k}_{r e f} \mathbf{e}_{x}\right)=\tau_{\mathrm{TE}}\left(\mathbf{k}_{t r n} \cdot \mathbf{e}_{x}\right)$

Note that $\left(\mathbf{k} \times \mathbf{e}_{\|}\right) \cdot\left(\mathbf{e}_{x} \times \mathbf{e}_{\|}\right)=\left(\mathbf{k} \cdot \mathbf{e}_{x}\right)$ for each of the plane waves. Also note that $\left(\mathbf{k}_{r e f} \mathbf{e}_{x}\right)=-\left(\mathbf{k}_{\text {inc }} \cdot \mathbf{e}_{x}\right)$. The well known solution of the above equations is

$\rho_{\mathrm{TE}}=\left(\mathbf{k}_{i n c} \cdot \mathbf{e}_{x}-\mathbf{k}_{t r n} \cdot \mathbf{e}_{x}\right) /\left(\mathbf{k}_{i n c} \cdot \mathbf{e}_{x}+\mathbf{k}_{t r n} \cdot \mathbf{e}_{x}\right)$

$\tau_{\mathrm{TE}}=2 \mathbf{k}_{i n c} \cdot \mathbf{e}_{x} /\left(\mathbf{k}_{i n c} \cdot \mathbf{e}_{x}+\mathbf{k}_{t r n} \cdot \mathbf{e}_{x}\right)$
The interesting scenario of total internal reflection results when $\mathbf{k}_{t r n} \cdot \mathbf{e}_{x}$ is pure imaginary. In that case, the coefficients are complex-valued, with $\left|\rho_{\mathrm{TE}}\right|=1$, as it must.

By contrast, consider the case of real $\mathbf{k}_{t r n} \cdot \mathbf{e}_{x}$, and the criterion that Equation (2), time-averaged Poynting flux, in the $\mathbf{e}_{x}$ direction, must be conserved. This leads to the following condition,

$\mathbf{k}_{i n c} \cdot \mathbf{e}_{x}-\left|\rho_{\mathrm{TE}}\right|^{2} \mathbf{k}_{i n c} \cdot \mathbf{e}_{x}=\left|\tau_{\mathrm{TE}}\right|^{2} \mathbf{k}_{t r n} \cdot \mathbf{e}_{x}$

where the common factor of $1 / 2 \omega / \mu_{0}$ has been removed, and of course, $|\psi(\tau(\mathbf{x}, t))|^{2}=1$ for real $\mathbf{k}$. We see that this equation is indeed satisfied with the above coefficients. This is an important observation for later work in the discrete problem, as it hints that Poynting conservation might serve as an alternate constraint to field continuity, for determining the scattering coefficients.

\subsubsection{TM Scattering in Continuum}

In the TM case, refer back to Equation (1) for the waveforms, set $\alpha_{T E}=0$ for all waves, set $\alpha_{T M}=1$ for the incident wave, and seek $\alpha_{T M}=\rho_{T M}$ for the reflected wave and, $\alpha_{T M}=\tau_{T M}$ for the transmitted wave. The solution over the entire spatial domain will then be of the form

$$
\begin{aligned}
& \mathbf{E}_{\text {analytic }}(\mathbf{x}, t)=\operatorname{Real}\left\{\left(\mathbf{e}_{\|} \times \mathbf{k}_{\text {inc }}\right) \psi\left(t_{\text {inc }}(\mathbf{x}, t)\right) S_{\text {inc }}(\mathbf{x})\right. \\
& \quad+\rho_{\mathrm{TM}}\left(\mathbf{e}_{\|} \times \mathbf{k}_{r e f}\right) \psi\left(t_{\text {ref }}(\mathbf{x}, t)\right) S_{r e f}(\mathbf{x}) \\
& \left.\quad+\tau_{\mathrm{TM}}\left(\mathbf{e}_{\|} \times \mathbf{k}_{t r n}\right) \psi\left(t_{t r n}(\mathbf{x}, t)\right) S_{\text {trn }}(\mathbf{x})\right\} \\
& \mathbf{B}_{\text {analytic }}(\mathbf{x}, t)=\operatorname{Real}\left\{\left(\omega \mu_{0} \varepsilon_{\text {inc }}\right) \mathbf{e}_{\|} \psi\left(t_{\text {inc }}(\mathbf{x}, t)\right) S_{\text {inc }}(\mathbf{x})\right. \\
& \quad+\rho_{\mathrm{TM}}\left(\omega \mu_{0} \varepsilon_{r e f}\right) \mathbf{e}_{\|} \psi\left(t_{\text {ref }}(\mathbf{x}, t)\right) S_{r e f}(\mathbf{x}) \\
& \left.\quad+\tau_{\mathrm{TM}}\left(\omega \mu_{0} \varepsilon_{t r n}\right) \mathbf{e}_{\|} \psi\left(t_{t r n}(\mathbf{x}, t)\right) S_{\text {trn }}(\mathbf{x})\right\}
\end{aligned}
$$

As before, in-plane electric and magnetic field continuity provide the conditions

$$
\begin{gathered}
\left(\mathbf{k}_{i n c} \cdot \mathbf{e}_{x}\right)+\rho_{\mathrm{TM}}\left(\mathbf{k}_{r e f} \mathbf{e}_{x}\right)=\tau_{\mathrm{TM}}\left(\mathbf{k}_{t r n} \cdot \mathbf{e}_{x}\right) \\
\varepsilon_{i n c}+\varepsilon_{i n c} \rho_{\mathrm{TM}}=\varepsilon_{t r n} \tau_{\mathrm{TM}}
\end{gathered}
$$

which has the well-known result,

$\rho_{\mathrm{TM}}=\left(\varepsilon_{t r n} \mathbf{k}_{i n c} \cdot \mathbf{e}_{x}-\varepsilon_{i n c} \mathbf{k}_{t r n} \cdot \mathbf{e}_{x}\right) /\left(\varepsilon_{t r n} \mathbf{k}_{i n c} \cdot \mathbf{e}_{x}+\varepsilon_{i n c} \mathbf{k}_{t r n} \cdot \mathbf{e}_{x}\right)$

$\tau_{\mathrm{TM}}=2 \varepsilon_{i n c} \mathbf{k}_{i n c} \cdot \mathbf{e}_{x} /\left(\varepsilon_{t r n} \mathbf{k}_{i n c} \cdot \mathbf{e}_{x}+\varepsilon_{i n c} \mathbf{k}_{t r n} \cdot \mathbf{e}_{x}\right)$

Again, note that the Poynting conservation theorem, for real $\mathbf{k}_{t r n}$, this time for the TM-mode,

$\varepsilon_{i n c} \mathbf{k}_{i n c} \cdot \mathbf{e}_{x}-\left|\rho_{\mathrm{TM}}\right|^{2} \varepsilon_{i n c} \mathbf{k}_{i n c} \cdot \mathbf{e}_{x}=\left|\tau_{\mathrm{TM}}\right|^{2} \varepsilon_{t r n} \mathbf{k}_{t r n} \cdot \mathbf{e}_{x}$

is satisfied for the above scattering coefficients.

\subsection{Discrete Scattering Coefficients}

The interface lies on a full grid-plane, normal to the coordinate direction $\mathbf{e}_{x}$. This means that, for the Yee-cell spatial discretization, the in-plane electric field components, $E_{y}$ and $E_{z}$, lie exactly on said interface, and so the analysis can continue to impose electric field continuity. However, the in-plane magnetic field components, $B_{y}$ and $B_{z}$, do not exist on the interface in the Yee-cell arrangement, but rather are half a cell above or below it. Thus, magnetic field continuity no longer has any requisite meaning. To make up for the loss of this criterion, introduce conservation of finitedifference Poynting flux, Equation (4), as the second criterion. Before proceeding, define some useful factors,

$\#_{\text {inc }} \equiv \cos \left(1 / 2 \mathbf{k}_{i n c} \cdot \mathbf{e}_{x} \delta x\right)$ 
$p_{\text {ref }} \equiv \cos \left(1 / 2 \mathbf{k}_{\text {ref }} \mathbf{e}_{x} \delta x\right)=\ddot{p}_{\text {inc }}$

$\beta_{t r n} \equiv \cos \left(1 / 2 \mathbf{k}_{t r n} \cdot \mathbf{e}_{x} \delta x\right)$

An important observation is that these $\beta_{-}$factors constitute a second-order-in-cell-size departure from unity, since $\cos (s) \cong 1-1 / 2 s^{2}$ for small $s$.

\subsubsection{TE Scattering in Discrete Maxwell}

As before, with $\mathbf{e}_{E}=\mathbf{e}_{\|}$for all three waves, the electric field continuity constraint still applies, but now the finitedifference Poynting flux constraint is applied, giving

$1+\rho_{\mathrm{TE}}=\tau_{\mathrm{TE}}$

$\beta_{i n c} \mathbf{k}_{i n c} \cdot \mathbf{e}_{x}-\left|\rho_{\mathrm{TE}}\right|^{2} \nexists_{i n c} \mathbf{k}_{i n c} \cdot \mathbf{e}_{x}=\left|\tau_{\mathrm{TE}}\right|^{2} \nexists_{t r n} \operatorname{Real}\left\{\mathbf{k}_{t r n} \cdot \mathbf{e}_{x}\right\}$

The solution is

$\rho_{\mathrm{TE}}=\left(\not_{i n c} \mathbf{k}_{i n c} \cdot \mathbf{e}_{x}-\ddot{p}_{t r n} \mathbf{k}_{t r n} \cdot \mathbf{e}_{x}\right) /\left(\not_{i n c} \mathbf{k}_{i n c} \cdot \mathbf{e}_{x}+\beta_{t r n} \mathbf{k}_{t r n} \cdot \mathbf{e}_{x}\right)$

$\tau_{\mathrm{TE}}=2 \beta_{i n c} \mathbf{k}_{i n c} \cdot \mathbf{e}_{x} /\left(\boldsymbol{\beta}_{i n c} \mathbf{k}_{i n c} \cdot \mathbf{e}_{x}+\beta_{t r n} \mathbf{k}_{t r n} \cdot \mathbf{e}_{x}\right)$

These are the exact reflection coefficients for the discrete problem. They are identical to the continuum scattering coefficients except for the $p_{-}$factors. And since, these contain $2^{\text {nd }}$-order departures from unity, it follows immediately that discrete Maxwell is $2^{\text {nd }}$ order accurate when the dielectric interface is exactly on a full-grid plane.

\subsubsection{TM Scattering in Discrete Maxwell}

In this case, $\mathbf{e}_{H}=\mathbf{e}_{\|}$for all three waves. The same electric field continuity constraint as in continuum still applies, as does now the finite-difference Poynting criterion,

$\left(\mathbf{k}_{i n c} \cdot \mathbf{e}_{x}\right)+\rho_{\mathrm{TM}}\left(\mathbf{k}_{r e f} \cdot \mathbf{e}_{x}\right)=\tau_{\mathrm{TM}}\left(\mathbf{k}_{t r n} \cdot \mathbf{e}_{x}\right)$

$\varepsilon_{i n c} \ddot{P}_{i n c} \mathbf{k}_{i n c} \cdot \mathbf{e}_{x}-\left|\rho_{\mathrm{TM}}\right|^{2} \varepsilon_{i n c} \ddot{P}_{i n c} \mathbf{k}_{i n c} \cdot \mathbf{e}_{x}=$

$\left|\tau_{\mathrm{TM}}\right|^{2} \varepsilon_{t r n} \nexists_{t r n} \operatorname{Real}\left\{\mathbf{k}_{t r n} \cdot \mathbf{e}_{x}\right\}$

The solution is

$\rho_{\mathrm{TM}}=\left(\varepsilon_{t r n} \nexists_{t r n} \mathbf{k}_{i n c} \cdot \mathbf{e}_{x}-\varepsilon_{i n c} \beta_{i n c} \mathbf{k}_{t r n} \cdot \mathbf{e}_{x}\right) /$

$\left(\varepsilon_{t r n} \beta_{t r n} \mathbf{k}_{i n c} \cdot \mathbf{e}_{x}+\varepsilon_{\text {inc }} \oiint_{i n c} \mathbf{k}_{t r n} \cdot \mathbf{e}_{x}\right)$

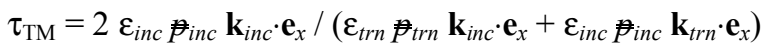

Again, this is identical to the continuum case, with $2^{\text {nd }}$ order correction due to the $\neq$ factors.

\section{IMPLEMENTATION OF FULL-FIELD/SCAT- TERED-FIELD FORMULATION}

A description of full-field/scattered-field implementation is well described and documented elsewhere, for example Reference [1]. In this section, we show the rather trivial generalization for the multiple waves due to scattering from the dielectric interface.

A key feature of this technique is that the sources are effectively currents, e.g., they add to the existing fields, rather than setting the field values directly. Thus any other waves, which might be present, simply pass through the locations of the sources unaltered. This gives rise to the fullfield (between the source-pairs)/scatter-field (outside the source-pairs) formalism.

Another key property is that with Yee-cell/leap-frog discretization, the technique can be essentially perfect to machine precision, even in $2 \mathrm{D}$ and $3 \mathrm{D}$, in particular, avoiding deleterious corner effects. In order for this to occur, the source-pairs must be half-a-cell apart, e.g., be adjacent $\mathbf{E}$ plane and H-plane components. Our implementation has followed figure 6.2 in Reference [1], with the inner source consisting of adjoining E-planes, and the outer source consisting of the surrounding B-planes. Thus, each E-field component has a surrounding B-field component, and these constitute a source-pair, as shown in Fig. (3). The analysis proceeds for each pair individually, with the only subtlety being that E-components on the edge of the box have Bcomponents in two directions, instead of just one. This is interpreted to be two separate source-pairs, and the resulting two co-located electric sources simply add together.

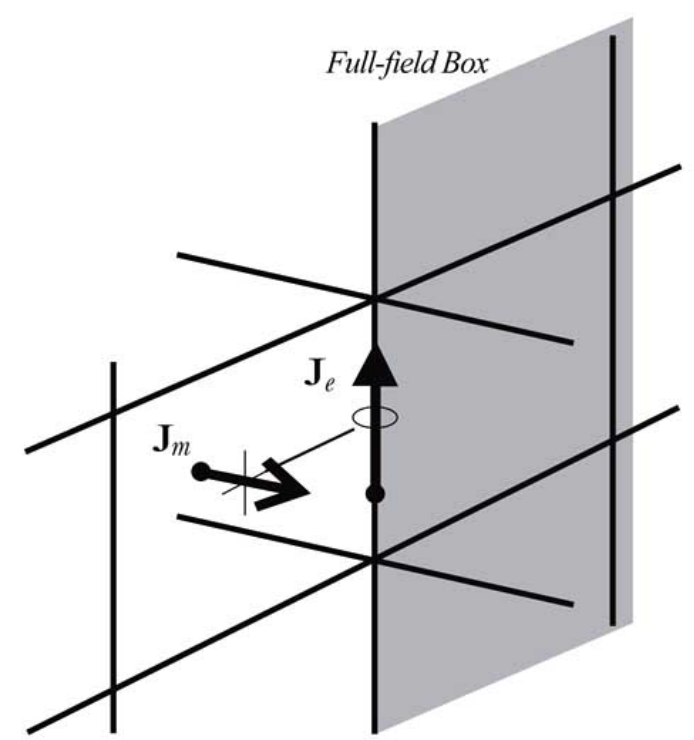

Fig. (3). Illustration of source pair in 3D. The full-field/scatteredfield approach can be made to be perfect, to machine precision, for Yee-cell/leap-frog discretization of Maxwell's equations, when the two sources are adjacent E and B components, separated by half-acell. The magnetic source, $\mathrm{J}_{\mathrm{m}}$, in particular, represents an artificial magnetic current term in Faraday's Law.

The algorithm proceeds as follows. Seek the electric and magnetic currents, $\mathbf{J}_{e}$, and $\mathbf{J}_{m}$, e.g., the source-pair, in the finite-difference (FD) discrete version of the Maxwell equations with sources, e.g.,

$\partial_{t, F D} \mathbf{B}=-\mathbf{J}_{m}-\nabla_{F D} \times \mathbf{E}$

$\partial_{t, F D} \mathbf{E}=-\varepsilon^{-1} \mathbf{J}_{e}+\left(\varepsilon \mu_{0}\right)^{-1} \nabla_{F D} \times \mathbf{B}$

These are:

$\mathbf{J}_{m}{ }^{n}=( \pm) \mathbf{E}_{\text {analytic }}\left(\mathbf{x}_{\mathbf{E}}, t^{n}\right) / \delta_{\mathbf{E} \times \mathbf{B}}$

$\mathbf{J}_{e}{ }^{n+1 / 2}=( \pm) \mu_{0}^{-1} \mathbf{B}_{\text {analytic }}\left(\mathbf{x}_{\mathbf{B}}, t^{n+1 / 2}\right) / \delta_{\mathbf{E} \times \mathbf{B}}$

where $\delta_{\mathbf{E} \times \mathbf{B}}$ is the grid size, e.g, $\delta x, \delta y$, or $\delta z$, in the direction running from the E-component to the B-component, and where $\mathbf{x}_{\mathbf{E}}$ and $\mathbf{x}_{\mathbf{B}}$ are the positions of the source-pair components in the Yee-cell centering scheme, where $\mathbf{E}_{\text {analytic }}$ and $\mathbf{B}_{\text {analytic }}$, are the analytic discrete representation waveforms given previously in Equations (8) and (12).

Note that in discrete Maxwell, the magnetic current is at the full-time step, and the electric current is at the half-timestep, and that the waveforms are evaluated at the corresponding time. However, the magnetic waveform is 
evaluated at the electric coordinate's position, half a cell away, and vice-versa for the electric current. This introduces the proper phase shift to get wave cancellation in one direction. The plus-or-minus signs are chosen so that the source-pairs emit or absorb radiation in the proper direction and sign. It is easy to see that if the discrete-E component matches $\mathbf{E}_{\text {analytic }}$, then the sign of $\mathbf{J}_{m}$ should be chosen so that it cancels the analogous quantity from the $\nabla \times \mathbf{E}$ term, in order to make the source-pair's discrete-B component maintain a zero value. And similarly, if the discrete-B component is zero, then the sign of $\mathbf{J}_{e}$ must provide the analogous missing quantity from the $\nabla \times \mathbf{B}$ term.

\section{DISCRETE IMPLEMENTATION AT THE SURFACE}

The interface plane between the dielectrics presents a special problem for a finite-difference implementation, due to the ambiguity in what to use for permittivity at the interface plane, $\varepsilon_{a v e, \|}$. This section serves to resolve this issue. Specifically, this permittivity is used to update the inplane electric field components, $E_{y}$ and $E_{z}$, in the discrete Ampere's law, as in

$E_{y}{ }^{n+1}-E_{\mathrm{y}}{ }^{n}=(\delta t / \delta x)\left(B_{z, \text { inc \& ref }}{ }^{n+1 / 2}-B_{z, t r n}{ }^{n+1 / 2}\right) /\left(\mu_{0} \varepsilon_{\text {ave, }}\right)$

where $B_{z, \text { inceref }}$ and $B_{z, t r n}$ are the magnetic fields half a cell below and above the interface plane. Also note that at the intersection of the dielectric interface and the full-field box, the same $\varepsilon_{a v e, \|} \|^{-1}$ multiplies the electric source, $\mathbf{J}_{e}$, from the source-pair, in Equation (21). The proper treatment of dielectric interfaces is an active area of research, see for examples Reference [5], however ours is a very simple geometry of a planar interface lying exactly on a grid line. It will be shown that this simplicity allows for an exact analytic result, even in the discrete case.

In general, one might expect that the $2^{\text {nd }}$-order accuracy of the Yee-cell will break down for the $\nabla \times \mathbf{B}$ operation which spans the interface, because $\mathbf{e}_{x} \cdot \mathbf{k}$ is not continuous across the interface, and so the finite-difference "derivative" is no longer well-centered. And furthermore this poorly-centered difference, which involves the transmitted wave on one side and the incident/reflected waves on the other, might be expected to produce an invalid electric field at the interface that does not match the analytic field. However, these expectations are overly pessimistic. In fact, the entire system remains $2^{\text {nd }}$-order accurate, even spanning the dielectric interface. A key point is that, in the discrete case, in-plane magnetic continuity at the interface has been relaxed. This means that the magnetic fields have limits, if extrapolated to the interface-plane, which do not match, from one side to the next. The discontinuity is inherent with the introduction of the $P_{-}$factors in the formulae of the scattering coefficients, Equations (18) and (20). We shall see that, remarkably, this discontinuity in magnetic field exactly counters the lack of well-centering.

To verify this assertion, first confine ourselves such that the incident half-space is for $x<0$, so that $k_{x, \text { inc }}=\mathbf{e}_{x} \cdot \mathbf{k}_{\text {inc }}>0$. Now consider the finite difference $\nabla_{F D} \times \mathbf{B}$ operation which crosses the interface, for the TE case, e.g.,

$$
\begin{gathered}
\delta x\left(\nabla_{F D} \times \mathbf{B}\right)_{\text {interface,TE }} \rightarrow k_{x, t r n} \tau_{\mathrm{TE}} \operatorname{Exp}\left(i^{1} / 2 k_{x, t r n} \delta x\right)- \\
k_{x, \text { inc }}\left(\operatorname{Exp}\left(-i^{1} / 2 k_{x, \text { inc }} \delta x\right)-\rho_{\mathrm{TE}} \operatorname{Exp}\left(i^{1 / 2 k_{x, i n c}} \delta x\right)\right)
\end{gathered}
$$

Outwardly, it appears that this difference is not wellcentered, due to the different wavenumbers, $k_{x, t r n}$ and $\aleph_{x, \text { inc }}$, in the arguments of the complex exponentials. However, plug in for the values of $\tau_{\mathrm{TE}}$ and $\rho_{\mathrm{TE}}$, from Equations (16) and (18), and perform manipulations to show that this is

$$
\begin{aligned}
& \delta x\left(\nabla_{F D} \times \mathbf{B}\right)_{\text {interface, } T E} \rightarrow \\
& \quad i \tau_{\mathrm{TE}}\left(k_{x, t r n} \sin \left(1 / 2 \xi_{x, t r n} \delta x\right)+k_{x, \text { inc }} \sin \left(1 / 2 k_{x, \text { inc }} \delta x\right)\right)
\end{aligned}
$$

Remarkably, the poorly-centered complex exponentials become centered, due to the specific form of the scattering coefficients, with the extra $\beta_{-}$factors of the discrete scattering-coefficients being critical to this algebra. In fact, this result is exactly half of the average of two well-centered differences, if each side had been analytically continued onto the other. Thus, one has

$$
\begin{aligned}
& \delta x\left(\nabla_{F D} \times \mathbf{B}\right)_{\text {interface }}= \\
& \quad 1 / 2 \delta x\left(\nabla_{F D} \times \mathbf{B}\right)_{\text {centered-incdref }}+1 / 2 \delta x\left(\nabla_{F D} \times \mathbf{B}\right)_{\text {centered-trn }}
\end{aligned}
$$

where it is noted that a similar process can be repeated with the TM polarization, with similar results. Each of the wellcentered $\nabla_{F D} \times \mathbf{B}$ 's will result in the correct analytic value of electric field, thanks to the imposition of in-plane electric field continuity. So with this knowledge, it is possible to produce the exact finite difference equation at the interface by adding together the following two well-centered equations,

$$
\begin{aligned}
& 1 / 2\left\{\varepsilon_{\text {inc }} \partial_{\mathrm{t}, \mathrm{FD}} \mathbf{E}_{\text {analytic }}=\mu_{0}^{-1}\left(\nabla_{\mathrm{FD}} \times \mathbf{B}\right)_{\text {centered-inc\&ref }}\right\} \\
& +1 / 2\left\{\varepsilon_{\text {trn }} \partial_{t, F D} \mathbf{E}_{\text {analytic }}=\mu_{0}^{-1}\left(\nabla_{F D} \times \mathbf{B}\right)_{\text {centered-trn }}\right\}
\end{aligned}
$$

$1 / 2\left(\varepsilon_{\text {inc }}+\varepsilon_{\text {trn }}\right) \partial_{\mathrm{t}, \mathrm{FD}} \mathbf{E}_{\text {analytic }}=\mu_{0}{ }^{-1}\left(\nabla_{\mathrm{FD}} \times \mathbf{B}\right)_{\text {interface }}$

which, due to the relation of Equation (26), results in a single equation, utilizing the actual interface difference. In comparing this to Equation (23), it is seen that the dielectric at the interface must then be the following,

$$
\varepsilon_{a v e, \|} \equiv 1 / 2\left(\varepsilon_{i n c}+\varepsilon_{t r n}\right)
$$

This result is consistent with a common-sense interpretation of the split-dielectric as two capacitances in parallel, as one would expect from the in-plane components.

We have verified this "exact" average dielectric for the interface, and the results are shown in Fig. (4). An effectively perfect full-field/scattered-field scenario is obtained, Fig. (4a, b), with noise levels around $-100 \mathrm{~dB}$, when using the exact value of Equation (28), which for this case is $\varepsilon_{a v e, \|} / \varepsilon_{0}=1.50$. These noise levels, visible on the logscale plot, Fig. (4b), are thought to be remnants of singleprecision round-off error, and show a noise-like spatial spectrum. Small changes to the value of $\varepsilon_{a v e, \|}$ are seen to result in noticeable failure of the full-field/scattered-field algorithm. In the example shown in Fig. (4c), a change in $\varepsilon_{a v e, /} / \varepsilon_{0}$ from 1.50 to 1.51 resulted in a visible signal outside the full-field box, of a planar nature, at the level of $-60 \mathrm{~dB}$ of the incident wave.

As an aside, we note that, when perfecting the implementation of the algorithm, it was often useful to look at the pattern of the signal in the scattered-field region, since its origin and shape often indicate where an error might have occurred. For example, in the Fig. (4d), the simulation used the correct $\varepsilon_{a v e, \|}$ for the source-free Maxwell's equation, but 
a) Exact $\varepsilon_{\text {ave, }}$ (Linear Scale)

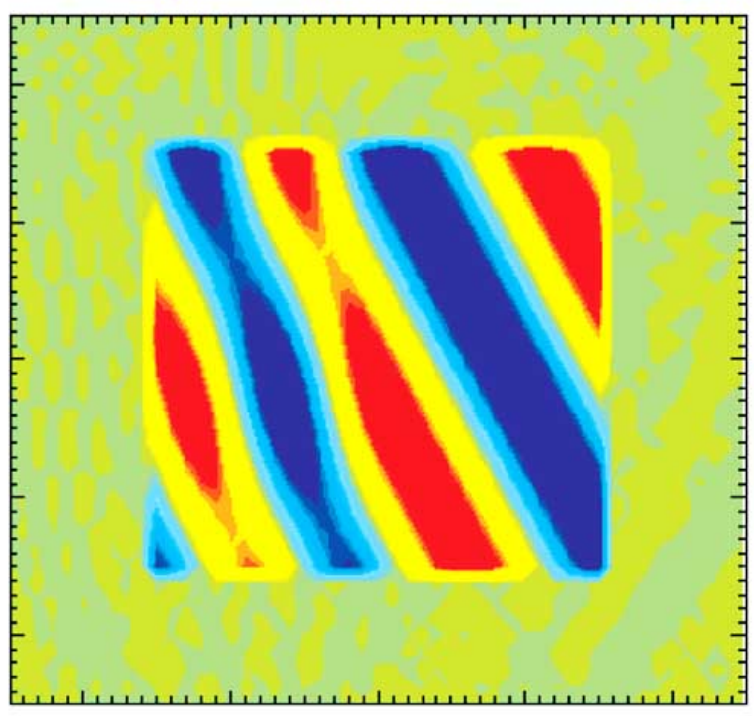

c) Non-Exact $\varepsilon_{\text {ave } \|}$ in Ampere's Law

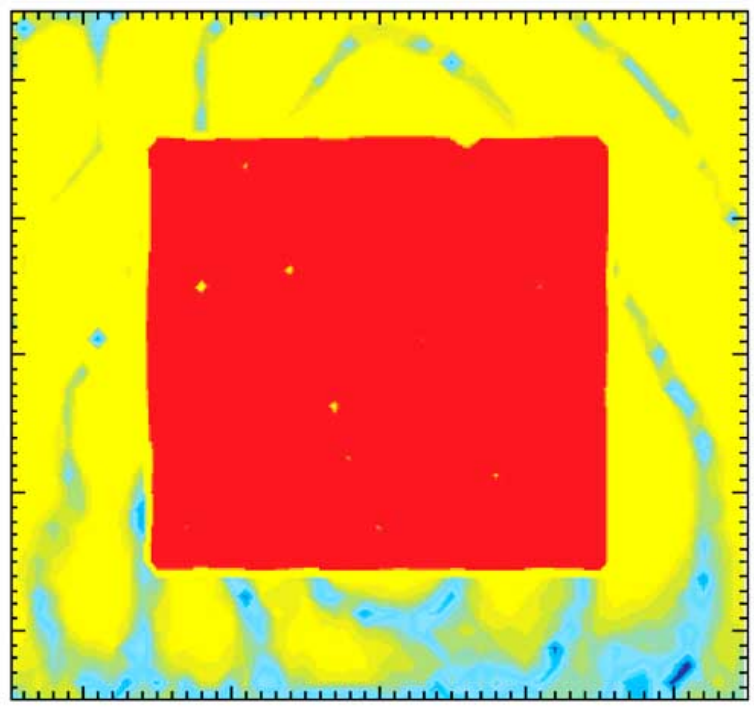

b) Exact $\varepsilon_{\text {ave, }}($ Log Scale)

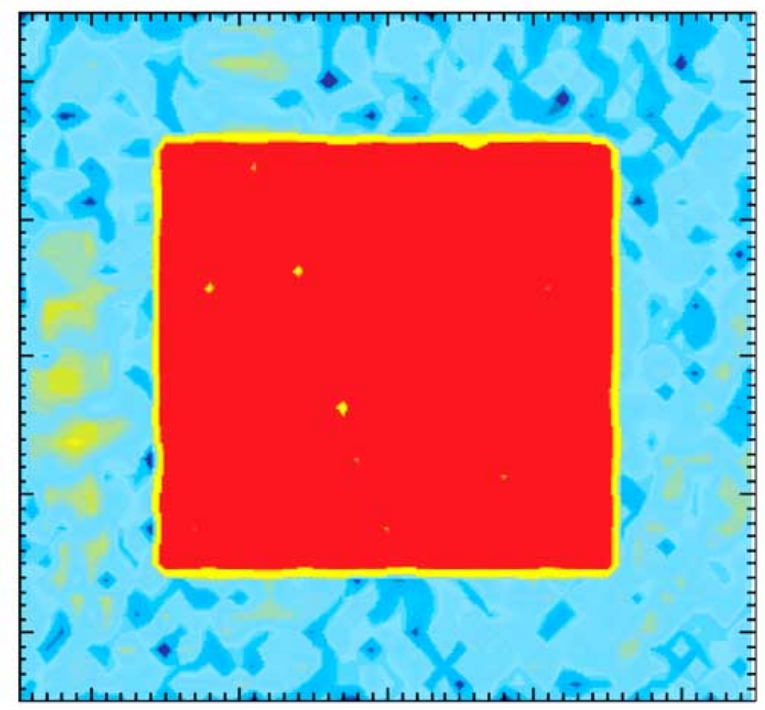

d) Non-Exact $\varepsilon_{\text {ave } \|}$ in Source

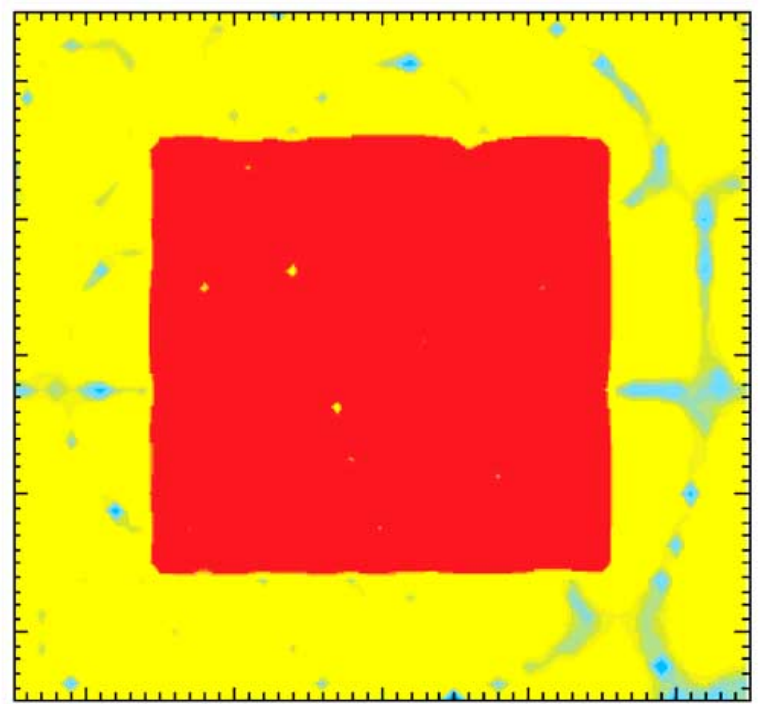

Fig. (4). Contour plots of $E_{z}$ field in TM case, for optimum and non-optimum values of $\varepsilon_{\text {ave, }, \|}$. When the optimum value is used, plots (a) and (b), the field in the outer scattered-wave region is $-100 \mathrm{~dB}$ down from the full-field, and shows random structure typical of floating-point round-off error. However, in plot (c) if a non-optimum $\varepsilon_{\text {ave,| }}$ is used in Ampere's Law, $0.51 \varepsilon_{\text {inc }}+0.49 \varepsilon_{\text {trn }}$ in this case, the log plot clearly shows escaping reflected and transmitted waves of about a $-60 \mathrm{~dB}$ amplitude. If instead, in plot (d), a non-optimum $\varepsilon_{\text {ave, } \|}$ is used in just the source term, $2 \varepsilon_{\mathrm{inc}} \varepsilon_{\mathrm{trn}} /\left(\varepsilon_{\mathrm{inc}}+\varepsilon_{\mathrm{trn}}\right)$ in this case, the untrapped waves, appear to emanate from the juncture of the full-field box and the interface.

used an improper $\varepsilon_{a v e, \|}$ multiplying the source, $\mathbf{J}_{e}$. The result was a scattered signal, notably of a spherically expanding nature, rather than plane-wave nature, emanating from the points at the juncture of the full-field box with the dielectric interface.

Finally, it is remarked here, but not discussed in detail, that this work was repeated, to treat the situation where the dielectric interface passes through the midplane of a cell, rather than lying on a grid-plane, as was assumed for all prior discussion. In such case, in-plane magnetic continuity is imposed, but electric continuity must be abandoned. Exact results are again possible, however in that case, the average dielectric for the $E_{x}$ component takes on a value that is consistent with capacitance in series, rather than in parallel, indeed, exactly as one might have expected from the normal electric field component.

Similar exact treatment of a dielectric interface that cuts through a cell at arbitrary coordinate is discussed in the Appendix. For such a situation, there are two planes which require a non-trivial permittivity, and two curl-differences which cross the interface, and thus risk being poorlycentered. Furthermore, such a situation has neither magnetic nor electric continuity, so constraints such as imposing the relationship of Equations (27) are employed in the derivation. It is shown mathematically that, by continuing to use the parallel and series capacitance analogy for the 
permittivity of electric field components which split the interface, $2^{\text {nd }}$-order accurate scattering coefficients result, even for arbitrary coordinate of the interface. This is an important result in the efforts to accurately model more general dielectric discontinuity conditions, as it provides limiting constraints on the even more difficult problem of treating a dielectric interface that crosses as arbitrary angle, as well as position, in a $2^{\text {nd }}$-order manner. Clearly, an important extension of this present work, if it were possible, would be to treat such an arbitrary crossing interface within the numerically exact constraints of the full-field/scatteredfield approach. It is felt strongly that this is a topic worthy of future work.

\section{DEMONSTRATIONS}

This section describes simulations done to explore the full range of capabilities of the augmented fullfield/scattered-field technique with dielectric interface. One important new situation, with no prior analogue in previous full-field/scattered-field implementations, is total internal reflection, where the transmitted-wave must be "re-absorbed' by the source-pairs, despite being evanescent, rather than propagating. A more traditional situation is the placement of a perfectly electrically conducting (PEC) material within the box, with resultant scattering from the object. Similarly, a non-planar dielectric interface can be studied from the point of view of what is the effective scattering, vis-à-vis a perfect planar interface. Finally, a case where the dielectric is nonpassive, in this case a plasma, is shown. A plasma can be considered as a type of "meta-material" capable of relative permittivity less than unity, and so this provides a hint how such materials can be treated in general.

In all simulation figures shown in this paper, we have used $\delta x=1 \mathrm{~cm}$, and $\delta y=1.25 \mathrm{~cm}$, and $\varepsilon / \varepsilon_{0}$ 's between -0.5 and 4.0 , to insure that results are not dependent on unit aspect ratio grid, for example. This corresponds to anywhere from 6 to 20 cells per wavelength. At these moderate resolution levels, the discrete Maxwell corrections to light propagation are sufficient that quite noticeable phase error would occur from using continuum light dispersion, rather than the discrete version of such.

\subsection{Total Internal Reflection}

Total internal reflection occurs when the incident $k_{y}{ }^{2}+k_{z}{ }^{2}$ $\geq \omega^{2} \mu_{0} \varepsilon_{t r n}$. In this case, all of the derivations in this paper carry forward even if $k_{x, t r n}$ is imaginary, rather than real. For example, in Equation (3b), simply use the relation that $\sin ^{-1}(i \lambda)=i \sinh ^{-1}(\lambda)$, and in Equation (16), use the relation that $\cos (i \lambda)=\cosh (\lambda)$. The result is that in Equation (3a), the phase functions, $\varphi(\mathbf{x}, t)$, will be complex valued, and the waveforms, $\psi(\varphi(\mathbf{x}, t))$, will thus contain exponential rather than sinusoidal behavior. However, if properly substituted into the source-pair relations, the exponentially decaying wave is properly "re-absorbed" in the full-field/scatteredfield technique.

This is illustrated by the simulations depicted in Fig. (5), where the angle of incidence is varied to either side of the critical angle. The incident wave propagates in a dielectric medium with $\varepsilon / \varepsilon_{0}=3$, which transitions to air, $\varepsilon / \varepsilon_{0}=1$ at the interface. In Fig. (5a), the angle-of-incidence is slightly above the critical angle, and the transmitted wave profile has a positive curvature, indicative of an evanescent wave. The result is total reflection, and so the waveform on the incident side is now a standing wave pattern, rather than propagating plane waves. In Fig. (5c), the angle-of-incidence is slightly below the crucial angle, and the transmitted wave has negative curvature, indicative of a propagating wave. Propagation at the critical angle is shown in Fig. (5b). Here the transmitted wave profile is flat, presumably out to infinity. In practice, it can take a long simulation time to fill in such a flat field profile, since the group velocity in the $x$ direction is essentially zero.

\subsection{Structures within the Full-Field Box}

The traditional application of the full-field/scattered-field technique is to look at the scattered wave of an object placed within the full-field box. A near-field-to-far-field transformation of this scattered wave is frequently done, as described in Reference [1]. The augmentation of the technique to include a dielectric interface allows one to look at the scattered wave, independent of the known reflective quality of the overarching interface between the dielectrics. One situation which immediately comes to mind would be to determine the scattering from an object floating on the surface of a liquid.

Another interesting scenario is to determine the forward radiation due to an object close to a dielectric interface operating under total internal reflection. This situation is shown in Fig. (6). In such a case, radiation can "tunnel" to the object in proximity, and then due to the object shape, can radiate in a forward direction, even though, in the absence of the object, there would be total reflection.

A related scenario involves analysis of scattering due to imperfections of the dielectric interface. Fig. (7) shows a simulation where the dielectric interface contains a "notch". The radiation pattern from this notch represents the "incremental" scattering, and includes a $-28 \mathrm{~dB}$ forward scattered wave and a $-47 \mathrm{~dB}$ backscattered wave visible in the scattered-field region. Further analysis along these lines might provide scattering characterizations of surface smoothness, damage, or other inherent imperfection.

\subsection{Plasma and Meta-Materials}

A further generalization of the full-field/scatter-field simulation is possible to allow for dielectrics of permittivity less than one, $\varepsilon / \varepsilon_{0}<1$, and in fact, even having negative values. Materials with such dielectrics are generally known as meta-materials. In addition, plasmas also have effective dielectrics which may be less than unity. Specifically, an unmagnetized plasma has dielectric

$\varepsilon / \varepsilon_{0}=1-\omega_{p}{ }^{2} / \omega^{2}$

where $\omega_{p}$ is the plasma frequency, which is proportional to the square-root of density. If the density is high enough that $\omega_{p}^{2}>\omega^{2}$, then $\varepsilon$ is indeed negative, and any wave propagating into such an over-dense plasma is reflected, regardless of angle of incidence. This formula may be reversed, so that given a desired dielectric, one can compute the plasma density which would result in that value of $\varepsilon$,

$\omega_{p}^{2}=n e^{2} / m \varepsilon_{0}=\omega^{2}\left(1-\varepsilon / \varepsilon_{0}\right)$

A plasma dielectric model is implemented in our FDTD simulation using a leap-frog scheme. A new plasma-electric- 
(a) total internal reflection
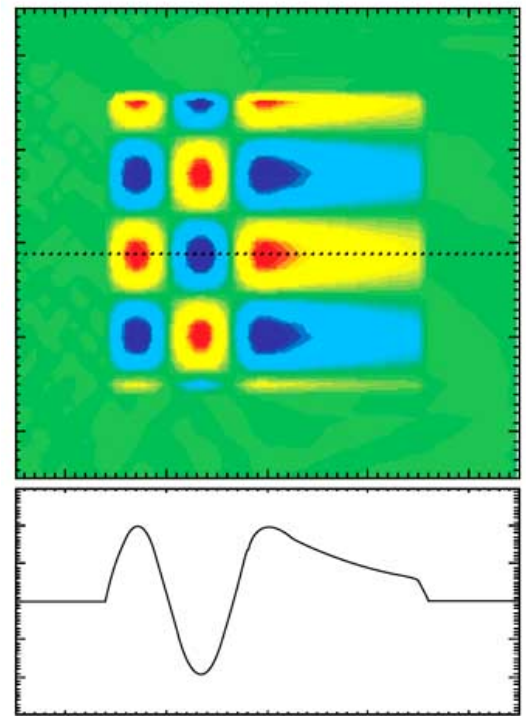

(b) at critical angle

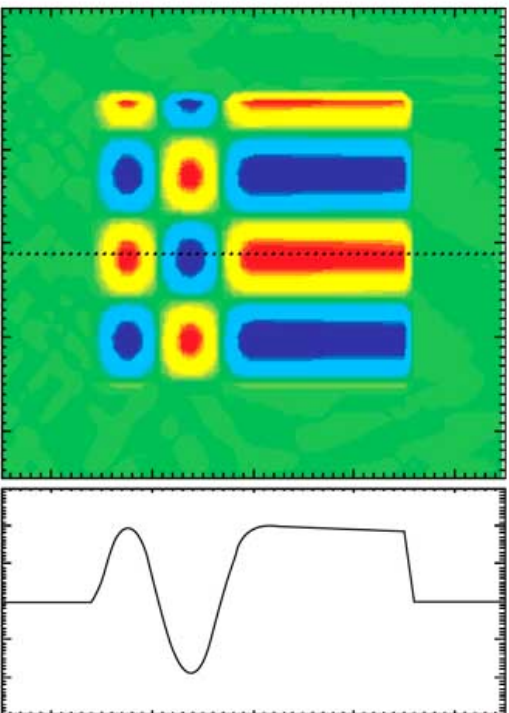

(c) transmitted wave

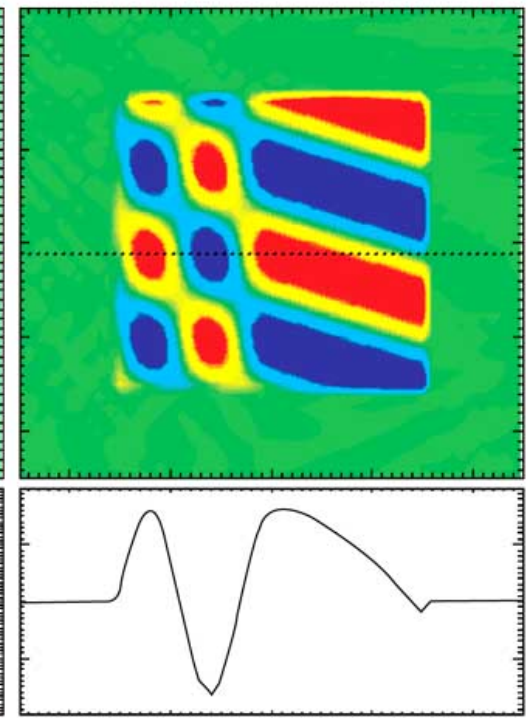

Fig. (5). The full-field/scattered-field algorithm works equally well in the case of total internal reflection, e.g., when the transmitted wave is exponentially decaying. Here, $\varepsilon_{\text {ind }} / \varepsilon_{0}=3$ and $\varepsilon_{\text {trn }} / \varepsilon_{0}=1$, with three different incident angles, $\mathrm{e}_{\mathrm{k}}=(0.8062,0.5916),(0.8165,0.5774)$, and $(0.8367,0.5477)$, corresponding to situations of total internal reflection, propagation at the critical angle, and a barely propagating transmitted wave. The field profiles through the dotted lines are shown.
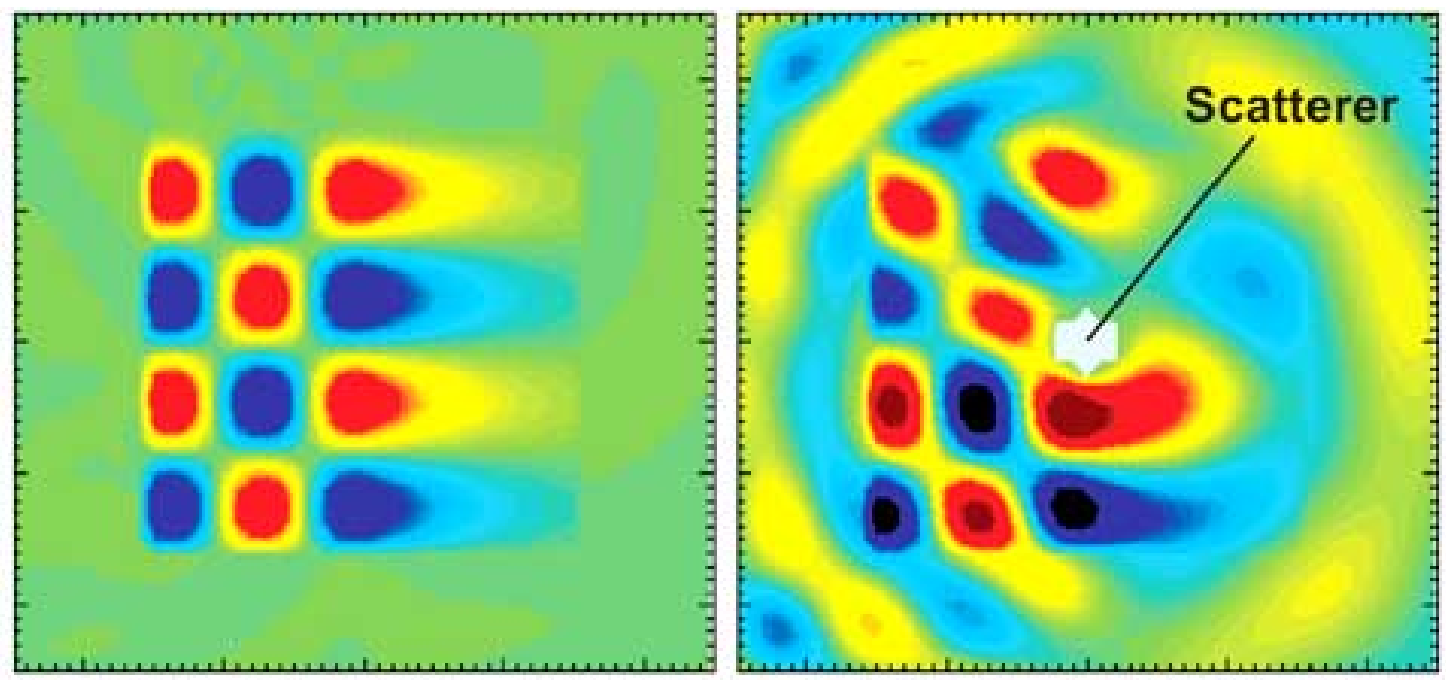

Fig. (6). Full-field/scattered-field simulation with and without a scatterer. The nominal simulation, on the left, is one of total internal reflection. A perfectly conducting quasi-spherical object is added (in gray), centered on the dielectric interface. This introduces a spherical scattered wave which is evident in the scattered-field region. Of note is that the scattered wave is propagating, rather than evanescent, on the transmitted side.

current field, $\mathbf{J}_{\text {plasma }}$, is introduced, which co-exists with the electric field on the Yee-cell arrangement, but is halfcentered on the time-steps. The update equation for this field is simply the finite-difference version of

$$
\partial_{t} \mathbf{J}_{\text {plasma }}=\omega_{p}{ }^{2} \varepsilon_{0} \mathbf{E}
$$

and this current is added into Ampere's Law in the normal manner. This implementation has the benefit that no reduction in time step is necessary, despite the fact that the phase velocity of light has increased due to $\varepsilon / \varepsilon_{0}<1$. However, there is a new stability criterion, $\omega_{p} \delta t<2$, which is easily met for all but the most extremely negative dielectrics. And in addition, the startup transient will excite a small amount of pure plasma oscillation, at a frequency near $\omega_{p}$, which radiates away rather slowly. Thus, simulations with this plasma model tend to require somewhat longer time-scales to arrive at the analytic CW state.

Fig. (8) shows two simulations using the plasma dielectric model. The first simulation is under-dense, with $\varepsilon_{\text {plasma }} / \varepsilon_{0}=0.5$, but with an angle that results in total internal reflection. Note how the evanescent longitudinal electric field on the plasma side, $E_{x, \text { plasma }} \sim E_{x, \text { inc }}\left(\varepsilon_{\text {inc }} / \varepsilon_{\text {plasma }}\right)$, is 


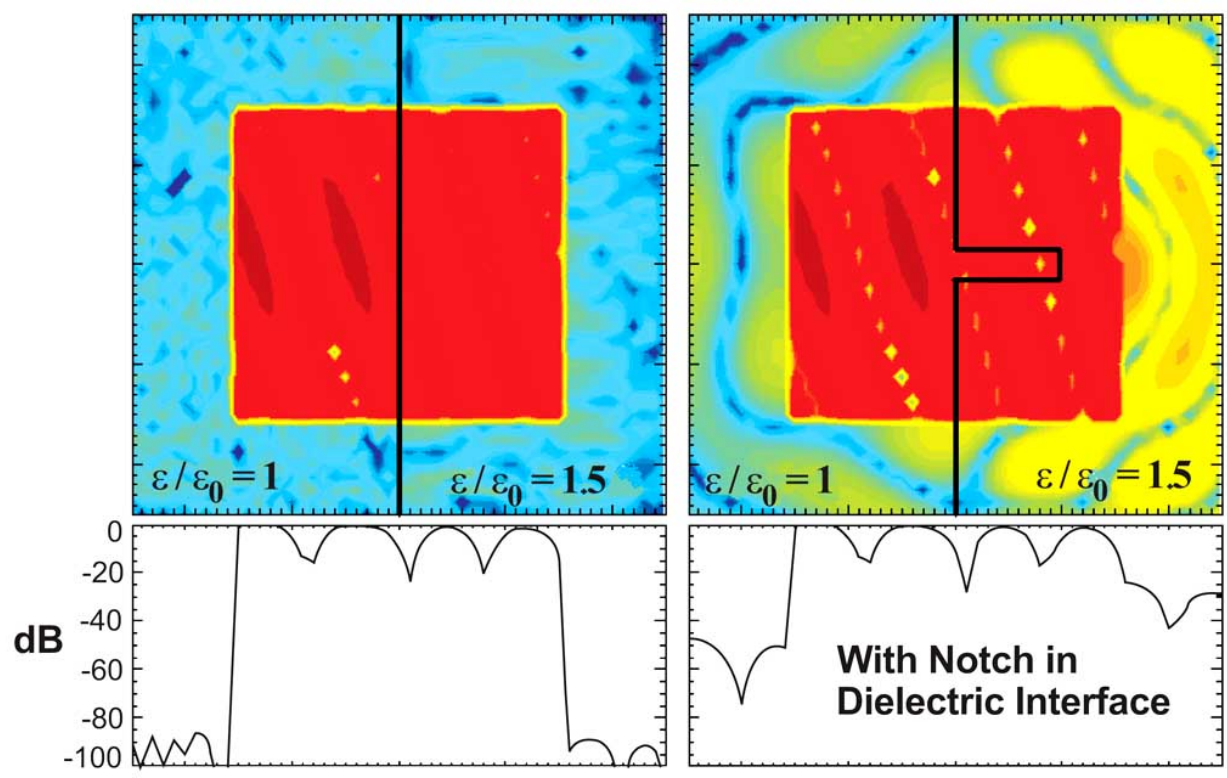

Fig. (7). Full-field/scattered-field simulation with notch in dielectric interface. Plots are log-scale, and profiles are through the midplane. The nominal simulation, on the left, is TE, and has $\varepsilon_{\text {ind }} / \varepsilon_{0}=1$ and $\varepsilon_{\text {trn }} / \varepsilon_{0}=1.5$, and a noise level around $-100 \mathrm{~dB}$. When a notch is introduced in the dielectric, a scattered wave is generated that radiates outside the full-field box. This scattered wave represents the "incremental" scattered wave due to the presence of the notch. It can be seen that amplitude of the forward scattered wave is approximately $-28 \mathrm{~dB}$, whereas the backscattered wave amplitude is approximately $-47 \mathrm{~dB}$.

enhanced by the smallness of $\varepsilon_{\text {plasma }}$. The second simulation is over-dense, with $\varepsilon_{\text {plasma }} / \varepsilon_{0}=-0.5$, which insures a very short-scale decay of the field on the plasma side.

More advanced plasma dielectric models, including implicit schemes that are not subject to the $\omega_{p} \delta t$ stability criterion, and magnetized plasmas that result in tensor dielectrics with additional internal resonances and cutoffs, are discussed in Reference [3]. Meta-materials will, in general, have some internal oscillation modes in analogy to the plasma oscillation, that allows for an effective dielectric constant less than unity. In some cases, one may simply replace the plasma frequency in Equation (31) with the
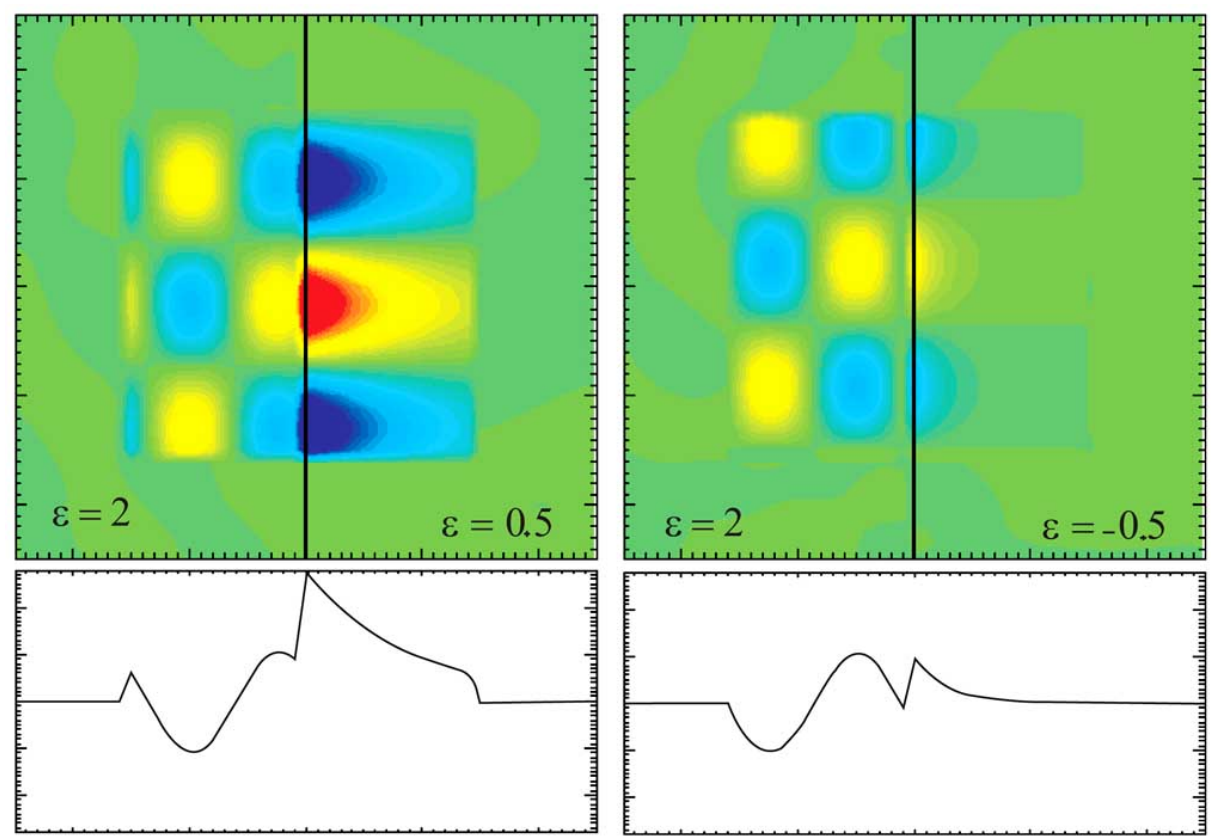

Fig. (8). Full-field/scattered-field simulation for a plasma dielectric. If $\varepsilon$ is less than unity, a plasma/meta-material can be invoked. Simulations are TM, and plots are of the longitudinal field, $\mathrm{E}_{\mathrm{x}}$. In the left figure, an under-dense plasma is reflecting due to angle of incidence. Note the large field increase which can be achieved on the plasma side, when $\varepsilon$ is small. In right figure, the plasma is over-dense (e.g., has $\varepsilon<0$ ), and all signals decay strongly. 
internal oscillation frequency of the meta-material to get a model of the meta-material. In other cases, the meta-material current may be magnetic in nature, rather than electric, or may have other subtle phasing aspects that require a more advanced model. In terms of future work, clearly this entire analysis can also be generalized to include non-vacuum permeability, $\mu \neq \mu_{0}$, in addition to $\varepsilon$, and also to include loss within these materials.

\section{CONCLUSIONS}

This paper has presented a generalization of the fullfield/scattered-field to include a planar dielectric interface within the simulation such that the nominal scattered and transmitted field from such an interface is captured at the full-field box, in analogous fashion to the incident wave. The original impetus driving this capability was the desire to have an effective plane-wave electromagnetic source in problems where the simulation domain is on the order of, or smaller than a single wavelength. Nevertheless, the augmentation of the technique is applicable to all of the usual full-field/scattered-field applications, including scattering-signatures, and meta-material research, and examples of such have been provided.

The implementation maintains the exact machineprecision quality of the traditional full-field scattered-field approach. This is accomplished with a careful analysis of the dielectric interface scattering coefficients in the case of the discrete representation. The discrete case scattering coefficients are provided as exact analytic expressions. An important aspect of this exact treatment is the verification of $2^{\text {nd }}$-order accuracy for the traditional series/parallel capacitance rules of effective permittivity for electric field components cut by the interface.

\section{APPENDIX A. DISCRETE SCATTERING COEFFICIENTS FOR ARBITRARY INTERFACE LOCATION}

It is possible to derive the analytic scattering coefficients for interface position anywhere along the $x$-axis, rather than requiring the interface to lie exactly on a grid plane. Introduce a new parameter, $h$, which represents the distance between the interface and nearest grid plane. A value of $h=0$ is the case treated in the main discussion, and in general, $h$ is constrained to the values, $-1 / 2 \delta x \leq h \leq 1 / 2 \delta x$. As was already noted in the discussion, the permittivity for the two in-plane electric field components whose areas are cut by the interface, $\varepsilon_{\text {ave }, \|}$, takes on an intermediate value between $\varepsilon_{\text {inc }}$ and $\varepsilon_{t r n}$. When $h \neq 0$, another intermediate permittivity, $\varepsilon_{a v e, \perp}$, must be introduced for the electric field component normal to, and whose length is cut by, the interface. That normal field component is to the left of the $\varepsilon_{\text {ave, } \|}$ for negative $h$, and to the right of $\varepsilon_{a v e, \|}$ for positive $h$. See Fig. (A1) for an illustration where the interface is shown at a negative value of $h$.

Permittivity, $\varepsilon$, has units of capacitance-length-per-area, and it is shown in this appendix that straight-forward serial/parallel capacitance rules provide the intermediatevalued permittivity, which do indeed result in $2^{\text {nd }}$-order accurate scattering coefficients, regardless of the interface location, $h$. We note that the following is an analytic result and thus carries greater certainty than a scaling study exhibiting $2^{\text {nd }}$-order accuracy, which is limited by practical time-constraint to finite choices over parameter space. Specifically, the intermediate-valued dielectrics are taken to be

$$
\begin{array}{ll}
\varepsilon_{\text {ave }, \|} & =1 / 2\left(\varepsilon_{i n c}+\varepsilon_{t r n}\right)+\left(\varepsilon_{i n c}-\varepsilon_{t r n}\right)(h / \delta x) \\
\varepsilon_{\text {ave }, \perp}{ }^{-1} & =\varepsilon_{ \pm}^{-1}+\left(\varepsilon_{i n c}{ }^{-1}-\varepsilon_{t r n}{ }^{-1}\right)(h / \delta x)
\end{array}
$$

where

$$
\begin{aligned}
\varepsilon_{ \pm} & =\varepsilon_{i n c}, \text { for }:-1 / 2 \delta x \leq h<0 \\
& =\varepsilon_{\text {trn }}, \text { for: } 0<h \leq 1 / 2 \delta x
\end{aligned}
$$

Note that the permittivity, $\varepsilon_{a v e, \perp}$, applies to different $E_{x}$ components, depending on the sign of $h$, and that it is not required, or defined, when $h=0$ exactly.

In the more general case of this Appendix, the derivation for the scattering coefficients is more cumbersome because neither electric nor magnetic field has a continuity condition between inc\&ref and trn-sides. But it is clear that any field component must take on the proper value associated with the side of the interface it is on, in order to correctly participate in finite-differences away from the interface. This implies that any differencing operation which spans the interface $\underline{\text { TE }}$

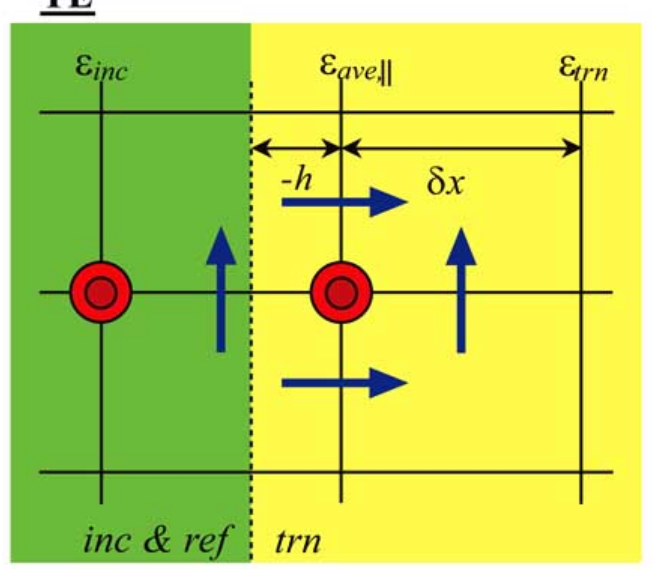

$\underline{\text { TM }}$

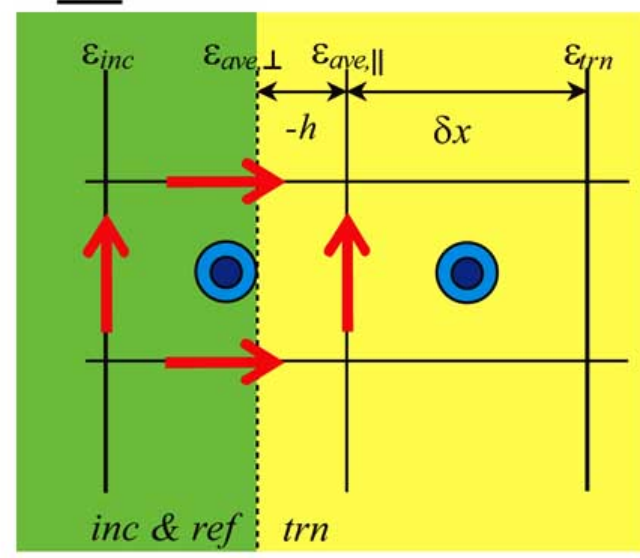

$\mathbf{E}$ in red

$B$ in blue

Fig. (A1). Illustration of dielectric interface a distance, $-\mathrm{h}$, from the nearest grid plane. There are no field continuity conditions, instead the operations which span the interface must result in the same values as if one-sided fields were analytically continued. 
between inc\&ref and trn-values, together with its material constant, must result in the same field update value, as if the proper one-sided fields were analytically continued to the other side. The field components involved in these interfacecrossing differences are illustrated in Fig. (A1), for the TE and TM cases, for a value of $h<0$.

Some simplification can be made by noting that the normal components are not involved in some of the curls, which then involve only the interface-crossing difference, which is denoted as $\nabla_{\perp}$,

$$
\begin{aligned}
\partial_{t} \mathbf{E}=\left(\mu_{0} \varepsilon\right)^{-1} \nabla \times \mathbf{B} & \Rightarrow \partial_{t} \mathbf{E}_{\|, \mathrm{TM}}=\left(\mu_{0} \varepsilon_{a v e, \|}\right)^{-1} \nabla_{\perp} \times \mathbf{B}_{\|, \mathrm{TM}} \\
\partial_{t} \mathbf{B}=-\nabla \times \mathbf{E} & \Rightarrow \partial_{t} \mathbf{B}_{\|, \mathrm{TE}}=-\nabla_{\perp} \times \mathbf{E}_{\|, \mathrm{TE}}
\end{aligned}
$$

Additional simplification can be made by noting that the updates for the normal components, that is $B_{x}$ for TE, and $E_{x}$ for TM, never involve interface-crossing differences, and thus these components always have phase associated with the in-plane dual-component that they surround. This allows the spatial-finite-difference of normal components to be lumped together with the temporal difference of the in-plane components as follows, leaving again, just the interfacecrossing difference,

$$
\begin{array}{ll}
\partial_{t} \mathbf{E}=\left(\mu_{0} \varepsilon\right)^{-1} \nabla \times \mathbf{B} & \Rightarrow \partial_{t} \mathbf{E}_{\|, \mathrm{TE}}=\left(\omega^{2} / k_{x, \text { ave, }{ }^{2}}\right) \nabla_{\perp} \times \mathbf{B}_{\|, \mathrm{TE}} \quad(\mathrm{A} 2 \mathrm{~b}) \\
\partial_{t} \mathbf{B}=-\nabla \times \mathbf{E} & \Rightarrow \partial_{t} \mathbf{B}_{\|, \mathrm{TM}}=-\left(\omega^{2} \mu_{0} \varepsilon_{\text {ave, },} / k_{x, \text { ave }, \perp}{ }^{2}\right) \nabla_{\perp} \times \mathbf{E}_{\|, \mathrm{TM}}
\end{array}
$$

where for algebraic convenience, define the following quantities

$$
\begin{array}{ll}
k_{x, \text { ave }, \|}{ }^{2} & \equiv \omega^{2} \mu_{0} \varepsilon_{a v e, \|}-k_{y}{ }^{2}-k_{z}^{2} \\
k_{x, a v e, \perp}{ }^{2} & \equiv \omega^{2} \mu_{0} \varepsilon_{a v e, \perp}-k_{y}{ }^{2}-k_{z}{ }^{2} \\
k_{x, \pm}^{2} & \equiv \omega^{2} \mu_{0} \varepsilon_{ \pm}-k_{y}{ }^{2}-k_{z}{ }^{2}
\end{array}
$$

$$
\begin{aligned}
& =k_{x, \text { inc }}{ }^{2} \text {, for: }-1 / 2 \delta x \leq h<0 \\
& =k_{x, \text { trn }}{ }^{2} \text {, for: } 0<h \leq 1 / 2 \delta x
\end{aligned}
$$

\section{A.1. TE}

Express the TE continuation conditions associated with the $\nabla_{\perp}$ operations of Equations (A2) in terms of the coefficients of Equation (8), assuming a negative value of $h$, as illustrated in Fig. (A1),

$$
\begin{aligned}
& \tau_{T M} \operatorname{Exp}\left(-i \xi_{x, t r n} h\right)- \\
& \left(\operatorname{Exp}\left(-i \xi_{x, i n c}(\delta x+h)\right)+\rho_{T M} \operatorname{Exp}\left(i \xi_{x, i n c}(\delta x+h)\right)\right) \\
& =\left(\operatorname{Exp}\left(-i \ldots_{x, i n c} h\right)+\rho_{T M} \operatorname{Exp}\left(i \ldots_{x, i n c} h\right)\right) \\
& -\left(\operatorname{Exp}\left(-i \hbar_{x, i n c}(\delta x+h)\right)+\rho_{T M} \operatorname{Exp}\left(i \hbar_{x, i n c}(\delta x+h)\right)\right) \\
& \left(1 / k_{x, a v e, \|}{ }^{2}\right)\left\{k_{x, \text { trn }} \tau_{T M} \operatorname{Exp}\left(i \ldots_{x, t r n}(1 / 2 \delta x-h)\right)\right. \\
& \text { - } k_{x, i n c}\left(\operatorname{Exp}\left(-i \xi_{x, i n c}(1 / 2 \delta x+h)\right)\right. \\
& \left.\left.-\rho_{T M} \operatorname{Exp}\left(i k_{x, \text { inc }}(1 / 2 \delta x+h)\right)\right)\right\} \\
& =\left(1 / k_{x, t r n}^{2}\right)\left\{k_{x, t r n} \tau_{T M} \operatorname{Exp}\left(i k_{x, t r n}(1 / 2 \delta x-h)\right)\right. \\
& \text { - } \left.k_{x, t r n} \tau_{T M} \operatorname{Exp}\left(-i k_{x, t r n}(1 / 2 \delta x+h)\right)\right\}
\end{aligned}
$$

For convenience define phase factors depending on $h$,

$\phi_{\text {inc }} \equiv \operatorname{Exp}\left(i\left(k_{x, t r n}-k_{x, i n c}\right) h\right)$

and $\phi_{r e f} \equiv \operatorname{Exp}\left(i\left(k_{x, t r n}+\hbar_{x, i n c}\right) h\right)$

And refer to Equation (3b) to define another useful P_factor,

$p_{\omega} \equiv \operatorname{sinc}(1 / 2 \omega \delta t)=\sin \left(1 / 2 k_{x, i n c} \delta x\right) /\left(1 / 2 k_{x, \text { inc }} \delta x\right)=$ $\sin \left(1 / 2 \hbar_{x, t r n} \delta x\right) /\left(1 / 2 k_{x, t r n} \delta x\right)$
With these definitions, the complex exponentials can be expressed algebraically in terms of the $\phi$ 's, $\not$ _factors, $k$ 's and $\delta x$. Solve for the scattering coefficient to get

$$
\begin{aligned}
& \rho_{T E}=\left(\phi_{i n c} / \phi_{r e f}\right)\left(\mathrm{A}_{T E}-\mathrm{B}_{T E}\right) /\left(\mathrm{A}_{T E}+\mathrm{B}_{T E}\right) \\
& \tau_{T E}=2 \phi_{\text {inc }} k_{x, i n c} \ddot{F}_{\text {inc }} /\left(\mathrm{A}_{T E}+\mathrm{B}_{T E}\right) \\
& \mathrm{A}_{T E} \equiv k_{x, \text { inc }} \not_{\text {inc }} \\
& \mathrm{B}_{T E} \equiv k_{x, \text { trn }} p_{\text {trn }}+i^{1 / 2}\left(k_{x, \text { inc }}{ }^{2}+k_{x, \text { trn }}{ }^{2}-2 k_{x, \text { ave }, \|}{ }^{2}\right) \delta x_{P_{\omega}} \\
& =k_{x, t r n} \nexists_{t r n}+i\left(k_{x, t r n}^{2}-k_{x, i n c}^{2}\right) h p_{\omega}
\end{aligned}
$$

where in the last form of the $\mathrm{B}_{T M}$ quantity, we have substituted the permittivity from Equation (A1). Equation (A3) of this calculation assumed that $-1 / 2 \delta x \leq h \leq 0$, but the calculation can be repeated for $0 \leq h \leq 1 / 2 \delta x$, with the same result as equation (A5), which is thus valid for the entire range of $h$ values. Recall that all $p_{-}$factors are $2^{\text {nd }}$-order, so any potential first order error terms will consist of those that involve $h$, which is present in $\phi_{i n c}$, $\phi_{\text {ref }}$, and $\mathrm{B}_{T M}$. But, it is easy to see that the lowest order $h$-terms cancel, such that the smallest error terms are $2^{\text {nd }}$-order in $h$, as well. Therefore, the scattering coefficients remain $2^{\text {nd }}$-order accurate, even for arbitrary crossing with $h \neq 0$. Note that the assumed form of $\varepsilon_{\text {ave,\|, }}$, in Equation (A1a) was key to achieving this result.

\section{A.2. TM}

Express the TM continuation conditions associated with the $\nabla_{\perp}$ operations of Equations (A2) in terms of the coefficients of Equation (8), assuming a negative value of $h$, as illustrated in Fig. (A1),

$$
\begin{aligned}
& \varepsilon_{\text {ave, } \|}{ }^{-1}\left\{\varepsilon_{t r n} \tau_{T M} \operatorname{Exp}\left(i \xi_{x, t r n}(1 / 2 \delta x-h)\right)\right. \\
& -\varepsilon_{\text {inc }}\left(\operatorname{Exp}\left(-i \xi_{x, \text { inc }}(1 / 2 \delta x+h)\right)\right. \\
& \left.\left.+\rho_{T M} \operatorname{Exp}\left(i k_{x, i n c}(1 / 2 \delta x+h)\right)\right)\right\} \\
& =\varepsilon_{t r n}^{-1}\left\{\varepsilon_{t r n} \tau_{T M} \operatorname{Exp}\left(i k_{x, t r n}(1 / 2 \delta x-h)\right)\right. \\
& \left.-\varepsilon_{t r n} \tau_{T M} \operatorname{Exp}\left(-i \xi_{x, t r n}(1 / 2 \delta x+h)\right)\right\} \\
& \left(\varepsilon_{a v e, \perp} / k_{x, a v e, \perp}{ }^{2}\right)\left\{k_{x, t r n} \tau_{T M} \operatorname{Exp}\left(-i k_{x, t r n} h\right)\right) \\
& \left.-k_{x, i n c}\left(\operatorname{Exp}\left(-i \hbar_{x, i n c}(\delta x+h)\right)-\rho_{T M} \operatorname{Exp}\left(i \hbar_{x, i n c}(\delta x+h)\right)\right)\right\} \\
& =\left(\varepsilon_{i n c} / k_{x, i n c}{ }^{2}\right)\left\{k_{x, i n c}\left(\operatorname{Exp}\left(-i{\aleph_{x, i n c}} h\right)-\rho_{T M} \operatorname{Exp}\left(i \ldots_{x, i n c} h\right)\right)\right. \\
& \left.-k_{x, i n c}\left(\operatorname{Exp}\left(-i \hbar_{x, i n c}(\delta x+h)\right)-\rho_{T M} \operatorname{Exp}\left(i \xi_{x, i n c}(\delta x+h)\right)\right)\right\}
\end{aligned}
$$

Define the following first order quantities,

$$
\begin{aligned}
\lambda_{\perp} & \equiv\left(\varepsilon_{a v e, \perp} k_{x, \pm}{ }^{2}-\varepsilon_{ \pm} k_{x, a v e, \perp}{ }^{2}\right) \delta x \\
& =\left(\varepsilon_{\text {inc }} k_{x, t r n}{ }^{2}-\varepsilon_{t r n} k_{x, i n c}{ }^{2}\right) h \\
\lambda_{\|} & \equiv\left(\varepsilon_{a v e, \|}-1 / 2 \varepsilon_{t r n}-1 / 2 \varepsilon_{i n c}\right) k_{x, i n c} k_{x, t r n} \delta x \\
& =\mathrm{k}_{\mathrm{x}, \text { inc }} \mathrm{k}_{\mathrm{x}, \mathrm{trn}}\left(\varepsilon_{\mathrm{inc}}-\varepsilon_{\mathrm{trn}}\right) \mathrm{h}
\end{aligned}
$$

The solution to the above equations is similar to the TE case, e.g., it can be written as

$$
\begin{array}{ll}
\rho_{T M} & =\left(\phi_{i n c} / \phi_{r e f}\right)\left(\mathrm{A}_{T M}-\mathrm{B}_{T M}\right) /\left(\mathrm{A}_{T M}+\mathrm{B}_{T M}\right) \\
\tau_{T M} & =2 \phi_{i n c} k_{x, i n c} \nexists_{i n c} /\left(\mathrm{A}_{T M}+\mathrm{B}_{T M}\right)
\end{array}
$$

However, now the forms for $\mathrm{A}_{T M}$ and $\mathrm{B}_{T M}$ differ slightly in the higher order terms, for the two cases, $-1 / 2 \delta x \leq h \leq 0$ and $0 \leq h \leq 1 / 2 \delta x$. For the case of Equation (A6), this is

For $-1 / 2 \delta x \leq h \leq 0$,

$\mathrm{A}_{T M}=k_{x, i n c} \varepsilon_{t r n} \nexists_{t r n}-i \nexists_{\omega} \lambda_{\|}$ 


$$
\begin{aligned}
& -1 / 2 \nexists_{\text {trn }} \ddot{P}_{\omega}^{2}\left(k_{x, i n c} \delta x\right) \lambda_{\perp}
\end{aligned}
$$

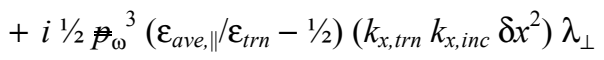

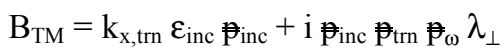

$$
\begin{aligned}
& + \text { Finc }_{\text {in }}{ }^{2}\left(\varepsilon_{\text {ave, } \|} / \varepsilon_{\text {trn }}-1 / 2\right)\left(k_{x, t r n} \delta x\right) \lambda_{\perp}
\end{aligned}
$$

A repetition of the calculation with the interface on the opposite side gives

For $0 \leq h \leq 1 / 2 \delta x$,

$$
\begin{aligned}
& \mathrm{A}_{T M}=k_{x, i n c} \varepsilon_{t r n} \nexists_{t r n}-i \nexists_{\omega} \lambda_{\|} \\
& -1 / 2 \nexists_{\text {inc }} \ddot{P}_{\omega}^{2}\left(k_{x, t r n} \delta x\right) \lambda_{\perp}
\end{aligned}
$$

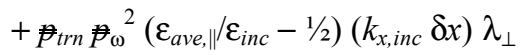

$$
\begin{aligned}
& +i \frac{1}{2} \nexists_{\omega}^{3}\left(\varepsilon_{\text {ave }, \|} / \varepsilon_{i n c}-1 / 2\right)\left(k_{x, i n c} k_{x, t r n} \delta x^{2}\right) \lambda_{\perp}
\end{aligned}
$$

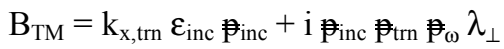

The first order terms are the same, regardless of the sign of $h$, and once again exactly cancel the first order terms in the $\phi$ 's, leaving scattering coefficients which are $2^{\text {nd }}$-order accurate, for any value of $h$. Again, the particular forms for $\varepsilon_{a v e, \|}$ and $\varepsilon_{a v e, \perp}$, in Equation (A1a), were key to achieving this result, as the $\lambda_{\|}$term of $\mathrm{A}_{T M}$ must combine properly with the $\lambda_{\perp}$ term of $\mathrm{B}_{T M}$, in order for the cancelation of first-order terms to occur.

The result of this Appendix is essentially a proof that:

- a dielectric interface parallel to one of the grid-planes, but cutting through the mesh at arbitrary coordinate normal to the interface, can be modeled in a $2^{\text {nd }}$-order accurate manner, and

- the permittivity which provides this $2^{\text {nd }}$-order accuracy is that which results from a common sense serial/parallel summation of capacitances where the dielectric interface cuts through the electric field element's length and areas.

Obviously, it would be nice to have some analogous proof for situations where the interface is at an arbitrary angle to the grid, rather than parallel to one of the gridplanes. This is a topic for further research. The fullfield/scattered-field approach developed in this paper can provide an excellent tool for testing and evaluation of possible techniques in this area, including the use of more general formulae for the $\varepsilon_{\text {ave }}$ 's. Furthermore, Equation (A1a) provides known $2^{\text {nd }}$-order accurate limit for these quantities, which must be met, when angle returns to parallel.

\section{REFERENCES}

[1] Taflove A. Computational electrodynamics the finite-difference time-domain method. Boston: Artech House, 1995; pp. 107-26.

[2] Yee KS. Numerical solution of initial boundary value problems involving Maxwell's equations in isotropic media. IEEE Trans Antenn Propag 1966; 14: 302-7.

[3] Smithe DN. Finite-difference time-domain simulation of fusion plasmas at radiofrequency time scales. Phys Plasmas 2007; 14: 056104.

[4] Barker RJ, Luhmann NC, Booske JH, Nusinovich GS. Modern microwave and millimeter-wave power electronics. Wiley-IEEE Press 2005; pp. 507-87.

[5] Werner GR, Cary JR. A stable FDTD algorithm for non-diagonal, anisotropic dielectrics. J. Comput Phys 2007; 226: 1085-101. 